\title{
Corela
}

Cognition, représentation, langage

13-2 | 2015

Vol. $13, n^{\circ} 2$

\section{Les prépositions évaluées par le prisme du paradigme cognitif : vers une lecture enactive}

\section{Aurélie BARNABÉ}

\section{OpenEdition}

\section{Journals}

Édition électronique

URL : http://journals.openedition.org/corela/4111

DOI : 10.4000/corela.4111

ISSN : 1638-573X

Éditeur

Cercle linguistique du Centre et de I'Ouest - CerLICO

Référence électronique

Aurélie BARNABÉ, «Les prépositions évaluées par le prisme du paradigme cognitif : vers une lecture enactive », Corela [En ligne], 13-2 | 2015, mis en ligne le 01 décembre 2015, consulté le 19 avril 2019. URL : http://journals.openedition.org/corela/4111 ; DOI : 10.4000/corela.4111

Ce document a été généré automatiquement le 19 avril 2019

\section{(c) (i) (2)(2)}

Corela - cognition, représentation, langage est mis à disposition selon les termes de la licence Creative Commons Attribution - Pas d'Utilisation Commerciale - Partage dans les Mêmes Conditions 4.0 International. 


\title{
Les prépositions évaluées par le prisme du paradigme cognitif : vers une lecture enactive
}

\author{
Aurélie BARNABÉ
}

\section{Introduction}

1 La langue, ensemble de systèmes de représentation, est un enchaînement mental et sémantique général. Le discours, en tant que mise en œuvre de la langue, consiste pour le locuteur à exécuter un parcours spécifique et paramétré en vue de produire une construction cohérente reflétée par les formes sélectionnées. Le passage langue/discours représente une expérience mentale introvertie et réflexive capable de mettre en œuvre sa propre dynamique. La forme langagière recueillie est traditionnellement envisagée indépendamment des conditions incarnées de sa production motrice et de son appropriation perceptuelle par les partenaires engagés (Bottineau, 2012e : 74). Or, le fait langagier est un comportement avant tout vocal, incarné, collaboratif, interactif et normé.

2 Le présent article envisage d'étudier l'expérience des conditions matérielles de la verbalisation. Il s'agit d'explorer la mise en œuvre de l'inscription expérientielle qui soustend la langue dans l'occurrence de prise de parole du sujet parlant. La présente étude est sous-tendue par l'hypothèse selon laquelle l'activité corporelle est un système d'action qui participe à la construction du sens, ce qui implique que l'émergence du sens non prédonné est inscrite dans la coordination de processus kinesthésiques. Notre hypothèse s'inscrit au croisement de deux paradigmes : le cognitivisme et "l'enactivisme $»^{1}$ (Varela et al. , 1993). Le cognitivisme renvoie à une conception spectatorielle du langage, conception dans laquelle s'inscrit le sujet parlant, observateur du monde pré-donné et muni d'un appareil psychologique représentationaliste encodée par des formes langagières (lexique, constructions etc.). L'enactivisme [de l'anglais to enact : susciter, faire émerger], en revanche, propose une théorie actantielle du langage où l'émergence du 
sens et des faits de conscience du sujet parlant est issue de coordinations corporelles incarnées, verbales et non verbales. L'approche de la perception par l'enaction envisage la cognition, non comme représentation, mais comme action incarnée (Thompson, 2007 : $13-22)^{2}$.

Le présent travail et l'hypothèse qui le sous-tend se basent sur le modèle de Talmy, dont les études, formulées suivant le paradigme cognitiviste, renvoient à des problématiques du courant enactiviste. Talmy représente sans doute le cognitiviste le plus intéressant pour les enactivistes, étant l'auteur de la «dynamique des forces » ou force dynamics - how entities interact with respect to force- with the exertion of force, resistance to such a force, blockage of the expression of force, removal of such blockage, and the like (Talmy, 2000a : 409). La dynamique des forces fait la part belle à la motricité et à la sensorialité de paramètres autres que visuels (multimodaux), même si Talmy les modélise de manière encore spectatorielle (en tant que cognitiviste). Le modèle syntaxique talmien propose en effet une approche dynamique de la syntaxe en linguistique cognitive, approche illustrée par la typologie langagière qui oppose les langues à cadrage verbal aux langues à cadrage satellitaire (Talmy, 2000a) : alors que les langues à satellites explicitent par le verbe le mode de déplacement (e.g. swim across the river) et donnent donc accès à la représentation d'un modèle de l'espace via une simulation motrice incarnée, les langues à cadrage verbal mettent en exergue l'objectif du déplacement (e.g. traverser la rivière à la nage), l'expression de l'objectif précédant celle de l'acte.

4 Les deux syntagmes verbaux juste cités illustrent la manière de se référer au même acte par le truchement de combinaisons langagières qui exemplifient deux types de processus de perception : le premier étant lié à l'expérience sensible du sujet parlant (cf. swim), le second mettant en avant la pensée théorique (cf. traverser), relayant la pensée pragmatique au second plan $^{3}$. Par leurs emplois verbaux, le français et l'anglais exemplifient respectivement ces deux profils typologiques. Par l'analyse de corpus oraux, réalisés dans le cadre d'une expérience recrutant 50 locuteurs francophones et anglophones, il s'agira, dans le présent article, de démontrer la mesure par laquelle l'activité motrice envisagée comme facteur permettant de promouvoir la construction du sens peut influencer la production langagière. Il sera question d'examiner les unités prépositionnelles employées par les locuteurs des deux langues, insérant ainsi notre étude dans une perspective contrastive.

5 Nous présenterons d'abord les enjeux théoriques de notre expérience, qui s'inscrit dans le cadre d'un protocole communément admis : celui de la typologie talmienne. Dans le cadre de l'expérimentation commentée, les descriptions françaises et anglaises recueillies font état de la verbalisation de mouvements d'entités variées dans l'espace. Les données des deux langues seront donc comparées et appréciées sous l'angle typologique talmien. L'expérience s'avère dépasser l'aspect strictement linguistique de l'évaluation qu'elle impose puisqu'une dimension non-linguistique s'inscrit dans les variables de l'expérience, dimension se rapportant au comportement moteur du sujet parlant et qui représente une contrainte à laquelle tous les locuteurs sont invités à se soumettre.

Dans un deuxième volet du présent article, on cherchera alors à comprendre l'opération de construction sémantique que cette approche active au moment où elle survient dans la linéarité. Notre objectif consiste à évaluer l'incidence qu'a cette variable d'ordre sensorimoteur et kinesthésique sur les descriptions langagières requises. En premier lieu, nous envisageons d'appréhender l'appartenance typologique du français et de l'anglais dans les descriptions langagières requises une fois le discours installé en situation 
interactive. Les données linguistiques regroupées, examinées sous l'angle cognitif que suggère la lecture talmienne, révéleront ensuite le maintien ou non de l'appartenance typologique des deux langues. La lecture cognitive des descriptions enregistrées mettra en lumière des données manifestement associées à la variable non-linguistique de l'expérimentation.

Dans un troisième temps, un lien sera donc établi entre les comportements langagiers des locuteurs et l'expérience corporelle sensorimotrice effectuée en amont des descriptions examinées. On s'attachera à explorer l'influence mutuelle des deux phénomènes; l'un langagier, l'autre kinesthésique, ce qui placera notre argumentation à un deuxième niveau d'analyse, dissocié, sur ce plan, de la typologie talmienne et de ses considérations strictement linguistiques. On s'interrogera sur les signaux perçus et reçus par le corps des locuteurs et sur leur impact vraisemblable sur les effets de sens dévoilés par les sujets. Ces questionnements se déprendront des réflexions traditionnelles sur la langue, alors examinée sous l'angle de l'enaction, à l'aube d'un nouveau paradigme linguistique qui se distingue du modèle cognitif mais qui annonce, comme nous le constaterons, une complémentarité effective.

\section{Antécédents théoriques}

\section{1. Mises en scènes verbales du mouvement dans l'espace}

Les résultats de la présente expérience se basant sur la typologie talmienne, nous en rappelons les principaux postulats. La distinction que fait Talmy entre les langues à satellites et les langues à cadrage verbal pour séparer l'expression des mouvements spatiaux des deux types de langue, relève de leur mise en scène verbale bien distincte. La syntaxe anglaise énumère iconiquement l'ordre de mise en place de la représentation visuelle simulée là où le français l'inverse: The bird hopped into the room - L'oiseau entra dans la pièce en sautillant. Cette inversion permet la détection et l'implication d'éventuelles redondances en français: The bird flew into the room - L'oiseau entra dans la pièce (*en volant). Plus généralement, l'anglais privilégie l'explicitation d'une vision symptomatique, passant sous silence son évaluation considérée comme évidente; le français, à l'inverse, sous-entend le symptôme et exprime le diagnostic: She stalked into the room - Elle entra dans la pièce d'un pas décidé ${ }^{4}$. Par sa typologie, Talmy identifie ainsi l'organisation conceptuelle dissemblable de l'expression de mouvements spatiaux, qui caractérise les langues à cadrage verbal (l'espagnol, l'italien, le français, le turc, l'hébreu, le coréen, le japonais, le grec, quelques branches de la langue maya, le nez-percé et la langue caddo) dont le français fait partie, et les langues à satellites (l'anglais, l'allemand, le néerlandais, le russe, le mandarin, le chinois, les langues finno-ougriennes, l'ojibwa et le warlpiri) comme l'anglais ${ }^{5}$.

\section{2. Méthodologie : description de l'expérience}

Selon l'appartenance typologique d'une langue donnée, la fréquence et la distribution des items verbaux qui contribuent à décrire le mouvement spatial (désigné motion event par Talmy [Talmy, 2000a]), ainsi que la récurrence des satellites et prépositions des différentes langues varient. Le présent travail se concentre particulièrement sur les satellites et unités prépositionnelles français et anglais qui permettent aux locuteurs 
interrogés de décrire ${ }^{6}$ la navigation spatiale d'éléments animés ou inanimés, comme l'indiquent les consignes figurant dans le tableau $\mathrm{n}^{\circ} 1$ ci-après.

Les considérations théoriques de cette première partie nous permettront d'évaluer le maintien de l'appartenance typologique du français et de l'anglais, une fois que le discours des locuteurs est installé en situation interactive. Le présent examen des occurrences distributionnelles et de la fréquence des items prépositionnels contribuant à lexicaliser le phénomène de mouvement se basera exclusivement sur les corpus oraux recueillis en français et en anglais. Les sujets participant à cette expérience ont entre 18 et 25 ans. Les anglophones étudient la langue française à l'université et inversement, permettant ainsi d'homogénéiser l'approche théorique des deux langues appréhendées par les sujets interrogés.

\section{2. 1. Expérience « statique»}

11 Les expériences présentées ci-après et les descriptions langagières qui en sont issues sont sous-tendues par l'hypothèse précédemment exposée selon laquelle l'activité corporelle est un système d'action qui participe à la construction du sens, ce qui implique que l'émergence du sens non pré-donné est inscrite dans la coordination de processus kinesthésiques. Selon cette hypothèse qui place la kinesthésie - en tant que variable non verbale - au centre des données langagières analysées infra, les deux expériences exposées en 2. 1. Expérience "statique » et 2. 2. Expérience "dynamique » renvoient à des états corporels statique et dynamique dont les sujets parlants font l'expérience, préalablement à la tâche de description langagière. Cette alternative corporelle s'avère nécessaire dans le cadre de l'expérimentation pour mettre au jour les résultats qui visent à confirmer ou infirmer notre hypothèse initiale.

Les données langagières recueillies font suite à la mise en œuvre d'une expérimentation partagée par deux étudiant(e)s, que nous nommerons «étudiant $\mathrm{n}^{\circ} 1$ » et «étudiant $\mathrm{n}^{\circ}$ 2 ». L'étudiant $n^{\circ} 1$, seul dans une pièce avec l'expérimentateur, écoute des consignes enregistrées et élaborées selon un protocole, lui donnant des instructions pour suivre un parcours composé de quatorze itinéraires. Suite à l'écoute des consignes, indiquées dans le tableau $\mathrm{n}^{\circ} 1$, l'étudiant $\mathrm{n}^{\circ} 2$ entre dans la pièce et a pour tâche de décrire l'itinéraire suivi par l'étudiant $n^{\circ} 1$.

Tableau $\mathrm{n}^{\circ} 1$ : Consignes de l'expérience

\begin{tabular}{|l|l|l|l|}
\hline Ordre $^{1}$ & $\begin{array}{l}\text { Chemin } \\
(\mathrm{s})^{2}\end{array}$ & total $^{3}$ & Instructions \\
\hline 1 & 1 & 1 & $\begin{array}{l}\text { Premièrement, vous allez marcher le long du chemin bleu et ramasser } \\
\text { deux balles de couleur différente. }\end{array}$ \\
\hline 2 & 1 & 2 & $\begin{array}{l}\text { Vous irez déposer ces balles dans le cerceau orange situé à côté du } \\
\text { parapluie. }\end{array}$ \\
\hline & 2 & $3-4$ & $\begin{array}{l}\text { Ensuite, vous reviendrez vous placer dans le cerceau qui marque le } \\
\text { point de départ et marcherez le long du chemin blanc. Vous } \\
\text { ramasserez de nouveau deux balles de couleur différente et }\end{array}$ \\
\hline
\end{tabular}




\begin{tabular}{|c|c|c|c|}
\hline & 1 & 5 & $\begin{array}{l}\text { Vous irez déposer ces balles dans le cerceau orange situé à côté du } \\
\text { parapluie. }\end{array}$ \\
\hline 3 & 2 & $6-7$ & $\begin{array}{l}\text { Ensuite, vous reviendrez vous placer dans le cerceau qui marque le } \\
\text { point de départ, puis vous marcherez le long du chemin jaune. Vous } \\
\text { choisirez un des deux ballons au bout du chemin. }\end{array}$ \\
\hline & 1 & 8 & Vous irez le déposer dans le cerceau situé à côté du parapluie. \\
\hline 4 & 2 & $9-10$ & $\begin{array}{l}\text { Vous reviendrez vous placer dans le cerceau qui marque le point de } \\
\text { départ puis vous marcherez le long des cordes et ramasserez deux } \\
\text { objets de votre choix qui se trouvent au sol. }\end{array}$ \\
\hline & 1 & 11 & Vous irez les déposer dans le cerceau à côté du parapluie. \\
\hline 5 & 1 & 12 & $\begin{array}{l}\text { A partir du cerceau à côté du parapluie, vous marcherez ensuite le } \\
\text { long du chemin orange, puis ramasserez un des deux frisbees au bout } \\
\text { du chemin. }\end{array}$ \\
\hline & 1 & 13 & Vous irez le déposer dans le cerceau à côté du parapluie. \\
\hline 6 & 2 & 14 & $\begin{array}{l}\text { Enfin, vous reviendrez au cerceau qui marque le point de départ. } \\
\text { Vous ramasserez ce cerceau et vous irez le déposer sur la table de } \\
\text { votre choix. }\end{array}$ \\
\hline
\end{tabular}

$13^{1}$ : Ordre des instructions $(1,2,3$, etc.).

$14^{2}$ : Nombre de chemins à suivre par consigne (1 ou 2).

$15^{3}$ : Nombre total de chemins à suivre au cours de l'expérience (14 au total).

16 La description que livre l'étudiant $n^{\circ} 2$ n'est donc pas précédée d'une expérience motrice mais d'une expérience corporelle statique, qui donnera lieu à ce que l'on classera parmi les « descriptions statiques ». On aura par ailleurs veillé à ce que l'étudiant $n^{\circ} 2$ occupe une position quasi-immobile pendant la première partie de l'expérimentation, qui correspond à l'écoute des consignes de l'étudiant $n^{\circ} 1$, en présence de l'expérimentateur.

\section{2. 2. Expérience « dynamique»}

17 L'étudiant $n^{\circ} 1$, en présence de l'expérimentateur et de l'étudiant $n^{\circ} 2$, écoute les consignes, lui donnant les directives pour suivre le parcours composé de quatorze itinéraires. L'étudiant $n^{\circ} 2$ écoute également les directives, ayant pour tâche d'observer l'étudiant $n^{\circ} 1$ et de reproduire le même parcours une fois que l'étudiant $n^{\circ} 1$ aura terminé le sien. L'étudiant $\mathrm{n}^{\circ} 1$ effectue donc son parcours. Une fois le trajet terminé, il lui incombe de décrire l'itinéraire suivi par l'étudiant $n^{\circ} 2$, qui lui, reproduit la série de chemins qu'il a vue l'étudiant $n^{\circ} 1$ parcourir. La description recueillie par l'étudiant $n^{\circ} 1-$ qui a eu l'expérience d'effectuer l'itinéraire composé de quatorze chemins avant de décrire la navigation spatiale de l'étudiant $n^{\circ} 2$ - correspond alors à une description précédée d'une expérience motrice, ce qui donnera lieu à ce que l'on classera parmi les « descriptions dynamiques ». 
18 La présente expérience propose donc aux participants de suivre un trajet, en leur imposant des contraintes similaires, dictées par un protocole analogue; certains étudiants proposant une description « statique » tandis que l'on recueille des descriptions «dynamiques » d'un second groupe d'étudiants. Dans un premier temps, le maintien de l'appartenance typologique du français et de l'anglais, une fois le discours des locuteurs installé en situation interactive, sera évalué. Dans un deuxième temps, l'expérience corporelle sensorimotrice statique ou dynamique précédant les descriptions langagières des sujets représente le trait à partir duquel sera relu le rattachement typologique des deux langues données, en fonction des comptes rendus recueillis.

\section{3. Prépositions et satellites}

\section{3. 1. Question terminologique}

19 La sémantique cognitive n'est pas une théorie du traitement des prépositions mais plutôt une recherche des conditions a priori de nos représentations du monde qui permettent l'emploi de ces prépositions. Ainsi, les présentes considérations théoriques sur les prépositions ne remettront pas en question les conditions d'applicabilité desdites unités, en revanche, nous insisterons sur la répartition de leur distribution et sur leur récurrence dans les deux langues considérées. De nombreuses études sur l'expression du " chemin » ou celle de la «trajectoire » (Slobin, 2003, 2004 ; Talmy, 2000a) font état des différences d'occurrence prépositionnelle en anglais et en français. Talmy insiste d'ailleurs sur les confusions éventuelles que peut susciter la distinction entre satellites et prépositions. Il définit les satellites comme suit:

It is the grammatical category of any constituent other than a noun-phrase or prepositional-phrase complement that is in sister relation to the verb root. It relates to the verb root as a dependent to a head. [...] English satellites largely overlap with prepositions - but together, apart, and forth, for example, serve only as satellites, while of, from, and toward serve only as prepositions. (Talmy, 2000b : 102)

20 To et over, par exemple, appartiennent aux deux catégories (Ibid. : 106-107) qui, selon Talmy, sont pourtant clairement définies :

Satellites should be well distinguished from prepositions [...] the two forms have quite distinct positional and grammatical characteristics. [...] However, a problem arises in English, which, perhaps alone among Indo-European languages, has come to regularly position satellite and preposition next to each other in a sentence. (Ibid $\therefore$ :106)

21 Les satellites se distinguent des adpositions dont Claude Hagège propose la définition suivante :

Adpositions (Adps) may be defined as grammatical tools which mark the relationship between two parts of a sentence: one is the element which an adposition governs. [...] English adpositions are prepositions, but other languages have postpositions. [...] Adps are the most frequent type of function marker. [...] Adps constitute, from the semantic and cognitive point of view, a complex set of interrelated meanings. (Hagège, $2010: 6$ )

Sur le plan syntaxique, les satellites dépendent des verbes avec lesquels ils entretiennent une relation privilégiée (in sister relation to the verb root [Talmy, 2000b : 102]), tandis que les adpositions introduisent des arguments (Grinevald et al. , $2011: 4$ ). Les racines lexicales des satellites peuvent être variées : satellites may be verbs, adverbs, preverbs, i.e. grammatical categories that have already been identified in languages (Ibid.: 2). Leur état de satellite est 
souvent dû à un processus de satellization: satellites constitute secondary systems, in the sense that they are the result of a process of satellization of elements already existing in the language ( Ibid:: 4). Sur le plan sémantique, les satellites font partie des unités lexicales qui contribuent à structurer le phénomène de mouvement.

Les unités lexicales, identifiées comme satellites dans une langue donnée, ont une incidence sur les considérations constructionnelles de cette langue. L'emploi de satellites détermine si la langue appartient aux S-framed languages ou aux V-framed languages. Talmy, pour classer une langue en tant que $S$-framed, regroupe, par ce terme, satellites et adpositions, ce qui représente une source de confusions puisque ces deux unités (satellite/ adpositions) sont syntaxiquement et lexicalement distinctes. Matsumoto (2011), à cet égard, identifie les langues $V$-framed de Talmy comme des head-framed languages, par opposition constructionnelle aux non-head framed languages, terme regroupant les satellites et les adpositions, qui correspond aux S-framed languages de Talmy (Ibid.: 3$)^{8}$. Par souci de précision terminologique, nous distinguons dans la présente étude, les adpositions des satellites dans la structuration linguistique du chemin. Nous nous référons aux unités spécifiques que regroupent ces deux termes: nous identifierons d'une part les prépositions (cf. adpositions) et d'autre part les particules, adverbes etc. (cf. satellites).

\section{3. 2. Verbalisation du phénomène de mouvement : conceptualisations multiples}

\subsection{1. Accumulation de prépositions}

Les langues à satellites ont en commun la facilité d'adjoindre plusieurs prépositions et satellites à un même verbe pour décrire un chemin complexe ${ }^{9}$ (Slobin, 1997, 2003, 2004), comme le souligne I. Ibarretxe-Antuñano citant Slobin :

Narrators in real narratives need not limit a path description to a single verb and its adjuncts [...] ; they may present a series of linked paths. (I. Ibarretxe-Antuñano, $2004: 328)$

Les descriptions des locuteurs anglais présentent effectivement ce type de chemins complexes :

(1) ${ }^{12} \mathrm{He}$ 's walking back across the room to the purple hoop along the orange cones. [ $\mathrm{AB}, 47]^{10}$

Dans l'occurrence (1), les prépositions across, to, along et le satellite back structurent le mouvement de l'entité humaine he que l'on désigne ici par le terme «figure » et que l'on opposera ultérieurement dans l'analyse au « fond $»^{11}$.

La combinaison de plusieurs prépositions et satellites à un même verbe, walk dans l'exemple juste cité, permet de conceptualiser le tracé du mouvement de la figure qui n'est pas nécessairement rectiligne comme le suggère le sémantisme des quatre items back, across, to et along. En aucun cas, nous n'assignons d'invariant sémantique pour interpréter le sens évoqué par les unités prépositionnelles juste citées, toutefois, la récurrence de leurs emplois en contexte et les études qui portent sur ces indices grammaticaux ${ }^{12}$, en l'occurrence back, across, to et along dans l'exemple (1), nous permettent d'en envisager un relief interprétatif relativement stable. C'est précisément au niveau de l'expression de ces chemins complexes, pour reprendre les termes de Slobin, qu'apparaît clairement la distinction entre langues à cadrage verbal et langues à cadrage satellitaire. 

dans les descriptions françaises recueillies, ce qui est imputable au schéma syntaxique français qui associe plusieurs prépositions beaucoup plus rarement que la langue anglaise pour segmenter les différentes voies dessinant un chemin :

Speakers of s-languages are more likely to break up the event into a larger number of components, based on 'narrative habits' of compacting several path components in a single clause. Speakers of V-languages, by contrast, have developed a narrative style that makes more sparing use of individual motion verbs to encode path components (Slobin, 1997 : 448).

Des chemins complexes restent toutefois employés en français et ces trajectoires verbales sont syntaxiquement admises.

\section{3. 2. 2. Boundary-crossing}

En ce qui concerne l'expression des chemins complexes, la différence majeure entre les deux groupes de langues se situe au niveau de la fréquence de leur emploi. Les occurrences (2) et (3) évaluent deux descriptions, le rapport du francophone interrogé (cf. [3]) étant comparé à celui du locuteur anglais (cf. [2]), les deux sujets décrivant leur partenaire au même stade du parcours :

(2) ${ }^{10}$ Now, she's going back towards the purple hoop along the blue path. [AB, 28]

(3) ${ }^{19}$ Elle fait marche arrière et retourne vers le cerceau violet, elle longe le chemin bleu pour regagner le point de départ. [FR, 7]

On note la mention de trois verbes en français (i.e. fait marche arrière, retourne et longe) là où un seul suffit en anglais pour tracer le même chemin (i.e. is going). La combinaison des prépositions towards, along et du satellite back au verbe go, en anglais, ne trouve pas de réciprocité syntaxique en français, puisqu'on ne dénombre qu'une seule préposition (i.e. vers):

Separate path verbs are used (...) and this is the required pattern in V-languages, because each of the events is a boundary-crossing event. (...) All direction specifications in a single simple clause referring to a single continuous motion event must denote the same 'unbounded' direction vector, i.e. the same direction vector. (Slobin [in Strömqvist \& Verhoeven], $2004: 246$ ) [nous soulignons]

Boundary-crossing renvoie au franchissement de frontière (Slobin, $2003: 10 ; 1997: 441)$ le long d'une trajectoire, dont la mention sollicite nécessairement un verbe différent pour une langue à cadrage verbal comme le français (cf. (3) fait marche arrière... retourne vers... longe) alors que ces franchissements peuvent être signalés par des satellites en anglais, associés au même verbe (cf. (2) going back...towards...along).

Cette divergence linguistique, dans l'organisation conceptuelle de l'expression de la navigation spatiale, implique immanquablement des disparités constructionnelles des deux langues ici examinées. Il s'agit, dans la partie qui suit, de mesurer les divergences syntaxiques qui caractérisent le français et l'anglais, en évaluant, dans un premier temps, l'ensemble des 50 descriptions recueillies. On appréciera le maintien ou non de l'appartenance typologique des deux langues, avant d'examiner, dans un deuxième temps, le maintien de ce résultat en se concentrant sur la variable non-linguistique de l'expérimentation, i.e. la posture corporelle. Les descriptions obtenues suite à l'expérience kinesthésique ${ }^{13}$ des sujets seront effectivement comparées aux rapports relevés du second groupe de locuteurs ayant eu une expérience corporelle quasiimmobile, en amont des discours recueillis. 


\section{Une typologie respectée}

\section{1. Fréquence des prépositions des modèles français et anglais}

À partir des descriptions requises auprès des locuteurs, on propose d'évaluer en priorité le paramètre constructionnel significatif lié à l'occurrence des prépositions dans les deux langues, c'est-à-dire la fréquence desdites unités, une fois le discours des participants installé en situation interactive.

Table 2 - Fréquence des occurrences prépositionnelles ${ }^{14}$ en français et en anglais

\begin{tabular}{|l|l|l|l|l|l|l|}
\hline & $\begin{array}{l}\text { Propositions } \\
\text { françaises }\end{array}$ & $\begin{array}{l}\text { Propositions } \\
\text { avec } \\
\text { prépositions }\end{array}$ & $\begin{array}{l}\text { Propositions } \\
\text { sans } \\
\text { préposition }\end{array}$ & $\begin{array}{l}\text { Propositions } \\
\text { anglaises }\end{array}$ & $\begin{array}{l}\text { Propositions } \\
\text { avec } \\
\text { prépositions }\end{array}$ & $\begin{array}{l}\text { Propositions } \\
\text { sans } \\
\text { préposition }\end{array}$ \\
\hline $\begin{array}{l}\text { Résultats } \\
\text { chiffrés }\end{array}$ & 515 & 242 & 273 & 474 & 353 & 121 \\
\hline$\%$ & $100 \%$ & $47 \%$ & $53 \%$ & $100 \%$ & $74,5 \%$ & $25,5 \%$ \\
\hline
\end{tabular}

Pour mener les différentes analyses qu'ont suscitées les descriptions, on a séparé chacune des propositions constituant les rapports des locuteurs. De fait, on obtient en français 515 propositions de la part des 25 locuteurs interrogés, comparé à 474 propositions en anglais d'un nombre similaire de participants. On observe un contraste manifeste dans les deux langues entre les propositions affichant au moins une proposition, i.e. $47 \%$ en français contre $74,5 \%$ en anglais. Ce résultat implique qu'une place lexicale saillante est attribuée aux satellites et prépositions dans l'expression consacrée au mouvement spatial en anglais. Effectivement, les occurrences semblables à l'exemple (4) sont relativement communes dans les descriptions anglaises :

(4) ${ }^{24}$ She's now walking back to the starting point along the green path. [AB, 43].

Cette occurrence comporte un satellite (i.e. back) et deux prépositions (i.e. to et along) qui se rapportent au même verbe, i.e. walk. En ce qui concerne les occurrences verbales, le nombre de propositions répertoriées dans chaque langue démontre que le français sollicite davantage de verbes conjugués, 515 exactement contre 474 pour les locuteurs anglais, dans les descriptions décrivant des phénomènes parfaitement analogues aux phénomènes observés par les anglophones, manifestations par ailleurs décrites par un nombre similaire de locuteurs qu'en français. Comme signalé précédemment (cf. 1.3.2.2), chaque franchissement de frontière (boundary-crossing) requiert l'emploi d'un verbe différent en français et il est difficile, dans l'organisation conceptuelle du mouvement spatial, d'associer plusieurs prépositions à un même verbe.

Les tableaux $\mathrm{n}^{\circ} 3$ et $\mathrm{n}^{\circ} 4$ représentent la répartition des items prépositionnels dans les deux langues : 
Table 3 - Occurrence des prépositions françaises dans les propositions incluant au moins une préposition

\begin{tabular}{|l|l|l|l|}
\hline $\begin{array}{l}\text { Propositions } \\
\text { françaises }\end{array}$ & $\begin{array}{l}\text { Propositions avec } \\
\text { préposition }\end{array}$ & $\begin{array}{l}\text { Propositions avec 2 } \\
\text { prépositions }\end{array}$ & $\begin{array}{l}\text { Propositions sans } \\
\text { préposition }\end{array}$ \\
\hline 514 & 239 & 3 & 272 \\
\hline $100 \%$ & $46.5 \%$ & $0.5 \%$ & $53 \%$ \\
\hline
\end{tabular}

En français, on observe un emploi presque unanime de propositions n'incluant qu'une seule préposition puisque seules trois unités propositionnelles (soit $0,5 \%$ des cas) contiennent deux prépositions. Ces résultats tendent à confirmer ce qu'impose le franchissement de frontière sur le plan syntaxique en français, qui recourt, semble-t-il, à davantage d'items verbaux, qui dénombrent une accumulation bien moindre de directions par le biais de prépositions, comparé à l'anglais, ce qui confirme l'une des conclusions auxquelles aboutit I. Ibarretxe-Antuñano, dans ses analyses de l'hypothèse de Dan I. Slobin: Concerning the description of motion vs. scene setting, V-languages devote less narrative attention to dynamics of movement (Ibarretxe-Antuñano, 2002: 11). Cette thèse s'appuie sur l'hypothèse de Slobin Thinking for Speaking ${ }^{15}$ :

According to this hypothesis, speakers of different languages attend to different components of motion events (Path, Manner, Figure, Ground) because their languages do not make these components equally salient. (Soroli et al. , 2012:4) Le français lexicalise le composant du chemin (path) par le verbe alors que l'anglais tend à réitérer cet aspect dans une même proposition par des emplois de satellites et de prépositions, comme le confirment les résultats exposés dans le tableau $\mathrm{n}^{\circ} 4$ :

Table 4 - Répartition des $74,5 \%$ de propositions présentant au moins une préposition dans les descriptions anglaises

\begin{tabular}{|l|l|l|l|l|l|}
\hline 1 préposition & 2 prépositions & 3 prépositions & 4 prépositions & 5 prépositions & 6 prépositions \\
\hline $43 \%$ & $41 \%$ & $7 \%$ & $3 \%$ & $3 \%$ & $3 \%$ \\
\hline
\end{tabular}

Sur les $74,5 \%$ de propositions contenant des satellites, on constate que ceux-ci sont employés suivant six schémas différents, incluant entre un et six satellites par propositions. Ce premier constat renforce le contraste entre français et anglais concernant la distribution occurrentielle des unités dans les deux langues. Le tableau $\mathrm{n}^{\circ} 4$ révèle un emploi très similaire de propositions n'incluant qu'un seul satellite ou une seule préposition (i.e. $43 \%$ ) avec les propositions en comportant deux (i.e. $41 \%$ ), comme dans l'occurrence (5) :

(5) ${ }^{25}$ She comes back again along the same diagonals. [AA, 44]

41 L'exemple (5) contient le satellite back et la préposition along. La combinaison de trois satellites s'avère plus rare (i.e. $7 \%$ ), et l'association de quatre, cinq ou six unités prépositionnelles l'est encore plus. On note que ces différentes accumulations de prépositions se manifestent à une fréquence identique : 
(6) ${ }^{14} \mathrm{He}$ 's walking back to the starting point along to the white line of white cones

towards the purple hoop at the end of the room. [AB, 50] syntaxique des trajectoires décrites par les locuteurs français et anglais -maintient l'appartenance typologique des deux langues, la distribution desdites unités, en revanche, soulève des questions d'ordre épistémologique qui ne relèvent pas de la typologie sur laquelle se base cette étude. Notons que l'hypothèse de Slobin, précédemment évoquée, est une version modifiée ${ }^{17}$ de l'hypothèse de Sapir-Whorf, selon laquelle les représentations mentales dépendent des catégories linguistiques, autrement dit, la façon dont on perçoit le monde dépendrait du langage. Cette forme de relativisme culturel appliquée au langage, dans la première moitié du XXe siècle, s'appuie sur des recherches typologiques et psycholinguistiques qui placent ce postulat au cœur d'une importante controverse de l'histoire de l'anthropologie cognitive. La présente expérience, centrée sur l'emploi des prépositions et satellites, fait écho au débat susmentionné par les résultats qu'elle met au jour ci-après.

\section{2. Les prépositions : entre conceptualisation, "subjectification ${ }^{18}$ " et « schématisation » d'un monde ${ }^{19}$}

\section{2. 1. L'orientation: une démarche culturelle}

Avant de faire état des hypothèses auxquelles donneront lieu les résultats issus des expérimentations précédemment décrites (cf. 1.2.1./1.2.2.), l'objet qui nous préoccupe nous oriente vers notre propre discours. C'est pourquoi nous proposons, dans un premier temps, une science de la démarche parallèle à une science de l'objet. La quête sémiotique 
des unités prépositionnelles dissimule le fondement de leurs types et de leurs limites. Different languages are sensitive to different properties, and they make different "choices» [...]. Languages categorize topological relations strikingly differently (Bowerman, 2007 : 177-). Les choix ("choices») dont fait état Bowerman font écho aux "preferences » précédemment évoquées par Ibarretxe-Antuñano.

L'emploi de prépositions dans la langue anglaise semble être imputable à une représentation du corps culturellement partagée. Selon Heine (Heine, 1997), le corps ne semble pas être perçu dans une position nécessairement verticale mais dans une posture penchée légèrement en avant (comme lorsque l'on marche). Cette hypothèse implique que la partie du corps head, par exemple, est communément interprétée par le biais des prépositions above et in front of tandis que buttocks s'associe avec below et behind (Heine, $1997: 57-64)$. Une autre conséquence de ce profil consiste à conceptualiser la partie supérieure et la partie inférieure du corps en contrepoint, comme le montre le modèle anthropomorphe de la Figure $n^{\circ} 1$ :

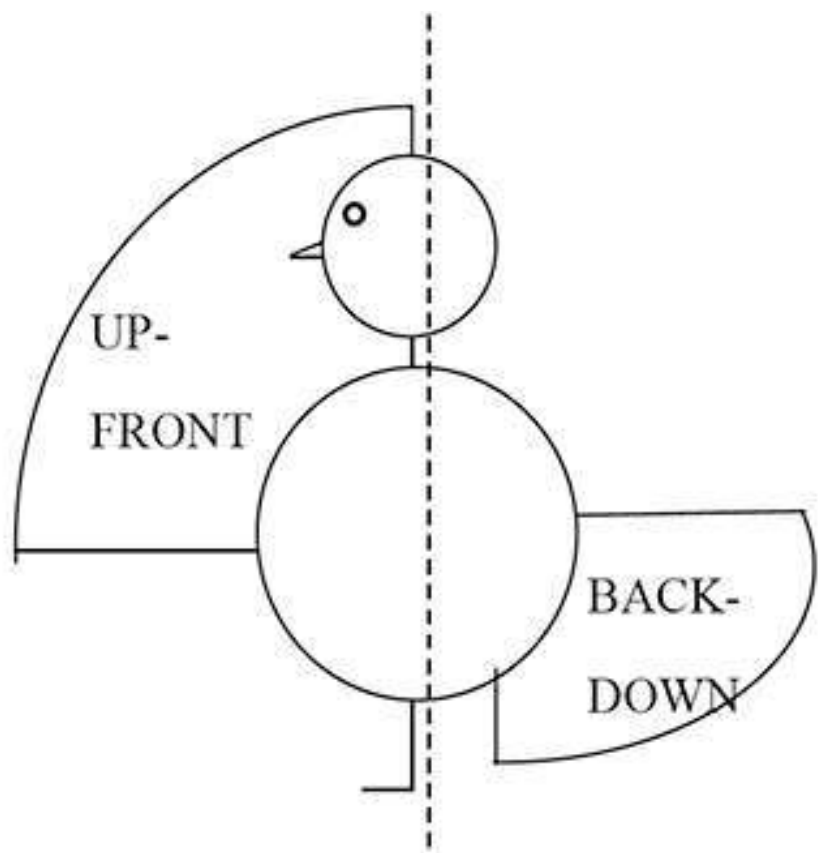

Figure $n^{\circ} 1$ : Points déictiques de l'orientation corporelle : profil anthropomorphe (Heine, 1997)

La Figure $\mathrm{n}^{\circ} 1$, sous-tendue par les observations de Heine, fait écho au commentaire de Langacker sur les recherches de Vandeloise centrées sur la spatialité et sur les prépositions: His [cf. Vandeloise] term anthropomorphic would seem to capture the essential unity of the various notions, which pertain to human interaction with the world at the physical, perceptual, and purposive levels. (Langacker, 2010 : \$5) [souligné dans le texte]. Ce constat concerne l'emploi des prépositions en anglais.

Des études identiques concernant la langue française donnent lieu à des conclusions analogues. Il existe, selon Borillo (Borillo, $2000: 259)$ plus d'une quinzaine de locutions prépositionnelles formées sur des noms de parties du corps en français moderne. Le français a d'ailleurs connu plusieurs grammaticalisation ${ }^{20}$ de mots désignant le flanc. On trouve ainsi dans la langue médiévale lez «à côté de » (du latin latus « flanc»), et coste "à côté de » (du latin costa) « côte, flanc », puis les constructions du costé de, au costé de, aux costés de, à costé de, formées en moyen français et en français classique sur costé (Fagard, 2012 : 3). Compte tenu de la récurrence des emplois prépositionnels en français et en 
anglais, outre la distinction typologique des deux langues, les remarques susmentionnées font état de la prise en considération synchronique de la représentation corporelle, référence qui a manifestement connu un développement diachronique régulier, notamment par le phénomène de grammaticalisation.

\section{2. 2. Une préposition : un point de vue}

Pour localiser deux entités par le biais d'une préposition, le sujet parlant n'évalue pas simplement la distance qui sépare les deux éléments : With projective adpositions [...], a third element is needed, i.e. a point of view (Herskovits, 1986:55). Si les prépositions véhiculées dans la langue anglaise font écho à la structure kinesthésique du modèle anthropomorphe précédemment cité, elles demeurent pour l'essentiel contextualisées, rappelle Svorou (Svorou, 1994: 20). Il identifie leur profil intrinsèque - «les cadres de référence » (reference frames) :

The notion which integrates observed behavior with respect to region assignment is the notion of reference frame (RF). This notion is fundamental in many theories of spatial relations with regards to projective relations. (Ibid. : 21)

51 Svorou distingue les cadres de référence "inhérents » (inherent $R F$ ), qui correspondent à des prépositions incluses dans des occurrences fournissant suffisamment d'informations spatio-temporelles pour en garantir une bonne réception interprétative par l'interlocuteur et les cadres de référence déictiques (deictic $R F$ ), dont l'interprétation des prépositions nécessite la présence du sujet parlant dans la scène donnée.

Le mouvement prend en compte la configuration géométrique d'un path-like object, pour reprendre les termes de Langacker (Langacker, $2000: 158$ ), dont l'orientation esquisse les représentations asymétriques avant-arrière. Ces repères spatiaux sont ceux d'une directionnalité inhérente au mouvement (Svorou, 1994: 184), dont le modèle anthropomorphe demeure la base de perception: The anthropocentric view of directionality constitutes the basis for the perception of direction of movement (Ibid. : 25). Les hypothèses de Svorou rejoignent la nécessite du point de vue que défend Herskovits: The fact that motion is understood through perception indicates that there is a point of view which provides a reference frame for specifying the directionality (Herskovits, 1986: 25).

Herskovits insiste sur la nécessité de situer la position spatiale d'une entité ( $\mathrm{x}$ ) par rapport à une autre entité $(\mathrm{Y})$, par le truchement d'une préposition. L'acte de localisation et celui de mouvement, tels qu'ils peuvent être identifiés par le biais d'une préposition ou d'un satellite rend compte de l'évaluation du locuteur de la distance qui sépare les entités à localiser, c'est-à-dire la figure et le fond ${ }^{21}$. Le chemin qui sépare (ou non) la relation figure/fond impose nécessairement une binarité dans le phénomène de localisation :

(7) ${ }^{18} \mathrm{He}$ 's going across the white line of cones. [AB, 37]

Svorou définit la représentation spatiale de across comme suit: Across - the landmark is treated as a stative entity with two boundaries; the trajector is treated as traversing the landmark from one boundary to the other (Svorou, 1994: 237).

\section{2. 3. Quel « accès conceptuel » aux prépositions ?}

Langacker, lui, appréhende l'apport sémantique des prépositions, insistant sur la portée psychologique qu'elles revêtent. La façon dont la préposition représente le temps implique les positions que la figure occupe au cours du temps comme saisies suivant un 
mode spécial de visée intellective, que Langacker baptise «inspection récapitulative » ( summary scanning); il définit ce processus comme suit :

Summary scanning: A mode of processing in which a series of component states are activated successively yet cumulatively ; thus, after a build-up phase, all facets of a complex structure are coactivated and simultaneously accessible. (Langacker, 1991 : 554)

La représentation graphique de la préposition through illustre ce processus cognitif dans la Figure $\mathrm{n}^{\circ} 2$. Par opposition à l'inspection récapitulative, on distingue le processus appelé « inspection séquentielle » (sequential scanning), que Langacker définit comme suit :

Sequential scanning: A mode of processing in which a series of component states are activated successively in non-cumulative fashion (i.e. a situation is followed in its evolution through conceived time, as in watching a film). (Ibid. : 553) La représentation graphique des prépositions from/to exemplifie l'inspection séquentielle dans la Figure $n^{\circ} 2$. L'inspection séquentielle et l'inspection récapitulative relèvent de l'imagerie conventionnelle, en ce sens que l'imagerie est constitutive du contraste sémantique que l'on trouve notamment en anglais, entre la préposition across et le verbe cross (Bottineau, $2010: 10$ ). Ces deux items diffèrent, non par le «contenu conceptuel » mais par le mode d'appréhension de ce contenu, c'est-à-dire par la nature de l'inspection mentale qui "accède» au concept (Langacker, 1987, dans 1991: 80, 132). En cela, la distinction entre ces deux unités est d'ordre sémantique.

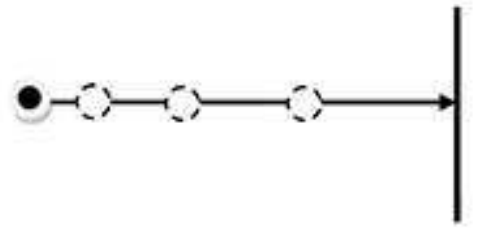

FROM/TO sequential scaming

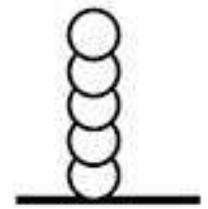

THROUGH stmmary scanning

Figure $n^{\circ} 2$ : Sequential scanning - Summary scanning distinguant notamment les prépositions " 1-way » des prépositions «2-way "22, l'étude des prépositions que fait Talmy précise l'accès conceptuel des locuteurs à certaines prépositions, ces explications identifiant également la complexité cognitive de cet accès, dont on constatera l'incidence effective dans les données analysées ci-après. Talmy explique que lorsqu'une entité en mouvement (i.e. la figure) est repérée par rapport à un point de référence (i.e. le fond), le premier élément fonctionne comme la figure psychologique (psychological figure), localisée en fonction de sa distance et de sa géométrie par rapport au fond psychologique (psychological ground). Chaque élément couvre un espace respectif, ce qui impose un partage biparti (bipartite partitioning) de la scène prise en considération (Talmy, 2000a : 313).

(8) ${ }^{19} \mathrm{He}$ 's going to the end of the room. [AB, 45]

Dans l'occurrence (8), l'entité he est considérée en fonction de son déplacement vers le fond the end of the room.

En revanche, certaines prépositions nécessitent la construction mentale d'un troisième espace (ou région), qui installe un partage triparti (tripartite scene partitioning)de la scène 
donnée. On doit alors considérer les deux entités à localiser (la figure et le fond) en fonction de ce troisième espace.

(9) ${ }^{22}$ And she's walking past the blue cones. [AB, 38]

61 Afin de repérer les entités mentionnées dans l'exemple (9), il incombe à l'observateur de la scène d'identifier un espace couvrant she et une autre région qui entoure the blue cones. En outre, il lui appartient d'identifier un espace contigu à blue cones à travers lequel il puisse situer le déplacement de she. Dès lors, l'interprétation de ce type de localisation implique la combinaison de la figure et du fond, qui constituent la figure psychologique ( psychological figure) tandis que la région, en arrière-plan (background), constitue le fond psychologique (psychological ground), comme le démontre la Figure $\mathrm{n}^{\circ} 3$ :

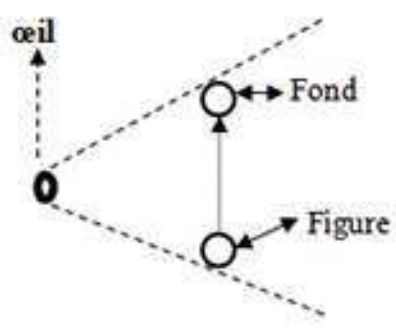

(40) répartition bipartie (cf. to)

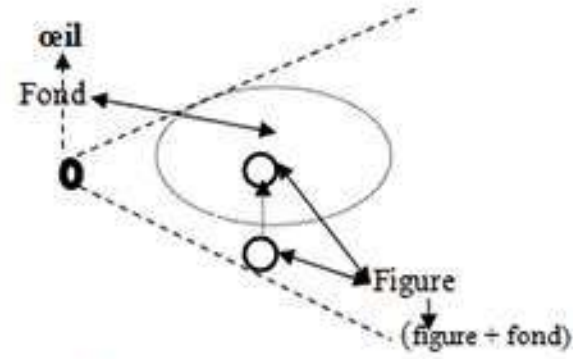

(41) répartition tripartie (cf. $B Q 5$, )

Figure $n^{\circ} 3$ :Répartition bipartie et tripartie de l'espace pour localiser la figure et le fond

Plusieurs prépositions présentent des distributions triparties en anglais comme en français (e.g. behind, between, entre, autour de etc.) dont nous analyserons celles insérées dans les données de l'expérience ici présentée.

\section{3. Distribution des prépositions}

\section{3. 1. Prépositions anglaises et françaises}

\section{3. 1. 1. Prépositions anglaises}

On a répertorié dans l'expérience toutes les prépositions et satellites qui ont permis aux locuteurs de faire état des occurrences de mouvement et de localisation.

Dans un premier temps, notre analyse vise à comparer les différentes unités employées par les locuteurs français et anglais, tous soumis aux mêmes contraintes expérientielles en amont des descriptions requises et astreints à évoquer des phénomènes similaires dans leur discours. Le tableau $\mathrm{n}^{\circ} 5$ expose les unités employées dans la langue anglaise, dont on rappelle que les données comptabilisent au total 474 propositions, comme l'indique le tableau $\mathrm{n}^{\circ} 2$ (cf. 2.1.). Le tableau $\mathrm{n}^{\circ} 4$ présente ici toutes les occurrences recueillies, sans faire, pour l'heure, de distinction entre les différents types d'expérience sensorimotrice imposés aux locuteurs en amont des descriptions sollicitées.

Table 5 - prépositions et satellites anglais ${ }^{23}$

\begin{tabular}{|l|l|l|l|}
\hline across & between & inside & past \\
\hline
\end{tabular}




\begin{tabular}{|l|l|l|l|}
\hline along & by & in the center of & through \\
\hline around & down & on & to \\
\hline at & from & out & toward \\
\hline at the end of & in/into & out of & up \\
\hline back & in between & over & via \\
\hline
\end{tabular}

On dénombre, au total, l'emploi de 25 prépositions et satellites pour faire état des actes de mouvement et de localisation, que l'on distingue comme suit :

(10) ${ }^{8} \mathrm{He}$ 's moving to the orange hoop at - at [sic] the end of the room. [AB, 33]

(11) ${ }^{19}$ She's stopping right in the center of the room. [AA, 29]

Les prépositions et satellites de chaque rapport des participants ont été pris en considération dans l'évaluation des données. En référence aux prépositions, qui présentent une schématisation tripartie, comme l'indique la figure $\mathrm{n}^{\circ} 3$, Talmy propose un classement desdites unités dont l'accès conceptuel impose une charge cognitive plus complexe que n'en requiert l'accès structurel des prépositions couvrant un partage biparti de la scène examinée pour situer la figure par rapport au fond. Le tableau $n^{\circ} 6$ présente les items prépositionnels en question :

Tableau 6 - Prépositions imposant une répartition tripartie de l'espace pour localiser la figure et le fond (Talmy, 2000A).

En comparant les unités du tableau $\mathrm{n}^{\circ} 5$ avec celles que propose Talmy dans le tableau $\mathrm{n}^{\circ}$ 6 , items intrinsèquement caractérisés par la complexité de leur accès conceptuel, on remarque que cinq d'entre eux sont employés par les locuteurs anglais dans leur description, à savoir :

around - between - down - in the center of - past

L'exemple (12) illustre cette division tripartie de l'espace pour situer la figure par rapport au fond:

(12) ${ }^{12}$ She's finally walking around the umbrella. [AB, 31]

En cette occurrence, il appartient à l'observateur de la scène d'identifier un espace qui couvre she et une autre dimension qui enveloppe the umbrella. Il lui incombe également de distinguer un espace attenant à the umbrella à travers lequel il puisse localiser le déplacement de she.

\section{3. 1. Prépositions françaises}

Dans la même veine, les 514 propositions françaises (cf. 2.1.) répertoriées présentent au total l'occurrence de 24 prépositions, comme l'indique le tableau n ${ }^{\circ} 7$ :

Tableau 7 - prépositions françaises

\begin{tabular}{|l|l|l|l|}
\hline à/au & au fond de & de & par \\
\hline
\end{tabular}




\begin{tabular}{|l|l|l|l|}
\hline à côté de & au milieu de & derrière & pour \\
\hline à droite de & au niveau de & du côté de & près de \\
\hline à gauche de & après & en direction de & vers \\
\hline à partir de & à travers & entre & parallèlement à \\
\hline au-dessus de & avec & jusqu'à & sur \\
\hline dans & autour de & le long de & \\
\hline
\end{tabular}

71 Parmi elles, cinq unités correspondent sémantiquement aux items identifiés par Talmy de par leur complexité d'accès, il s'agit des prépositions suivantes :

au milieu de - autour de - derrière - entre - le long de

72 L'exemple (13) exemplifie la division tripartie de l'espace à laquelle nous soumet l'emploi de la préposition derrière :

(13) ${ }^{17}$ Puis elle rejoint le cerceau orange derrière la ligne de plots bleus. [FR, 24]

73 À l'instar des exemples (9) et (12), des espaces adjacents à chaque entité nominale de l'occurrence (13) doivent être identifiés par l'observateur pour favoriser l'accès conceptuel de la précision sémantique de derrière.

74 On note que les prépositions qui apparaissent en italique dans le tableau $\mathrm{n}^{\circ} 7$ se rapportent à ce qu'Aurnague identifie comme des Noms de Localisation Interne (NLI), c'est-à-dire qu'elles permettent de se référer à diverses parties d'une entité spatiale (Aurnague, 2005: 1). Plusieurs analyses ont été consacrées au statut desdites unités, comme le rapporte Aurnague :

Certaines analyses (Aurnague 89) (Borillo A. 88) considèrent les NLI comme des substantifs alors que d'autres (Borillo A. 92) ${ }^{24}$ mettent plutôt l'accent sur leur caractère relationnel en montrant, notamment, le figement progressif des locutions prépositionnelles dérivées de ces marqueurs. (Ibid. : 1)

Effectivement, les items en italique du tableau $n^{\circ} 7$ n'apparaissent dans les données qu'en tant que locutions prépositionnelles ${ }^{25}$. En référence au statut des prépositions françaises, on observe que les données collectées sont composées de prépositions et de locutions prépositionnelles, comme mentionné supra; on ne retient en revanche aucune préposition issue d'anciens participes (e.g. touchant, nonobstant, excepté, supposé), aucune préposition en voie de disparition (e.g. deçà, delà, devers),ni aucune préposition proche de locutions prépositives comme quant $\grave{a}^{26}$.

Les unités les plus employées par les locuteurs interrogés concernent, pour la plupart, des items que Melis identifie comme "prépositions simple» à partir d'une liste de 26 prépositions qu'il propose ${ }^{27}$ (Melis, 2003: 105). Par prépositions «simples», nous entendons les plus typiques, comme le souligne Melis lui-même : " plus typiques, c'est-àdire les prépositions simples, qui apparaissent au locuteur comme inanalysables » (Ibid. : 105). Les prépositions les plus récurrentes dans les données correspondent effectivement à celles que Melis classe parmi les plus fréquentes. On retient notamment les occurrences de à, de, avec, pour, par et après. du tableau $\mathrm{n}^{\circ} 7$, il apparaît que les locutions prépositionnelles à gauche de et à droite de ne 
trouvent pas de réciprocité sémantique en français. Par ailleurs, la postérité spatiale, signalée par derrière en français, se manifeste par back en anglais, essentiellement employé en tant que satellite et pas en tant que préposition (e.g. ${ }^{5} \mathrm{He}$ 's coming back to the purple hoop. $[A B, 24])$. À ce propos, on note l'absence de la préposition behind en anglais pour indiquer la postérité spatiale, exclusivement indiquée par derrière en français. Nous n'approfondirons pas ici ces constats inter-langagiers dont il nous semble toutefois intéressant d'en recenser les disparités d'emploi comme celles susmentionnées.

\section{4. La préposition : motivation conceptuelle ou applicabilité linguistique disponible} réellement au cœur de la question de Sapir-Whorf, selon laquelle nous disséquons la nature suivant des lignes tracées d'avance par nos langues maternelles (Extrait de Cahiers de linguistique française). Cette thèse est elle-même issue de la conception humboldtienne concernant la paire langue/réalité, Humboldt pour qui l'homme vit en communion avec les objets, mais seulement à la manière dont la langue les lui présente. (Humboldt [1836/2001: 106] dans Paprocka-Piotrowska, 2012 : 145). Whorf renchérira ultérieurement :

Les usagers de grammaires nettement différentes sont orientés par leurs grammaires vers des observations de types différents et des évaluations différentes d'actes de perception extérieurement similaires, de ce fait, ils ne sont pas des observateurs équivalents et ils doivent forcément aboutir à des visions du monde quelque peu différentes. (Whorf, 1940/1982 : 297, dans Ibid. : 145) [nous soulignons]

Les remarques de Whorf s'accordent, en partie, avec les constats des emplois prépositionnels sémantiques nuancés en français et en anglais mentionnées précédemment ; en partie car Whorf centre son propos sur des perceptions différentes qui donneront lieu à des observations distinctes, issues d'usagers de « grammaires nettement différentes ». En ce qui concerne les disparités relevées entre le français et l'anglais, si nos remarques sont principalement d'ordre sémantique et se rapportent à l'appréhension conceptuelle des différents types de prépositions dans les deux langues, celles de Whorf se rattache davantage aux moyens dont dispose la grammaire d'une langue donnée.

81 Un parfait exemple pour illustrer « ces observations de types différents » est celui de la typologie, précisément discutée en première partie de cet article, qui identifie une attention variable des sujets parlants décrivant une scène de mouvement, selon qu'ils parlent une langue à cadrage verbal ou une langue à satellites (languages attend to different components of motion events (Path, Manner, Figure, Ground) because their languages do not make these components equally salient [Soroli et al. , 2012: 4]). L'applicabilité de cette typologie ayant été démontrée via les corpus oraux de la présente expérience, les deux langues analysées manifestent toutefois des divergences d'ordre sémantique quant à leur emploi 
respectif des items prépositionnels. Nous considérons ces différences indirectement liées aux hypothèses susmentionnées.

\section{Ouverture d'un débat épistémologique}

\section{1. Le sujet parlant : un sujet vivant}

82

cela a été démontré quant à la différence d'emploi de prépositions d'une même langue (cf. 2.2.3.), l'appréhension conceptuelle constitue une source de variation sémantique. Pour justifier les cas de variation, on a tendance à parler d'acception centrale, de prototype de la catégorie (Lakoff, 1987, appendice 2), de conceptualisation fondamentale (Ibid.) ou plus spécifique (Langacker, 1986b), de subjectification (Langacker, 1987) - autant de théories qui évaluent les moyens linguistiques dont disposent les usagers d'une langue pour transmettre leur perception et leur entendement d'une réalité vécue. L'orientation d'une sémantique qui tâcherait de décrire les principes cognitifs motivant la structure de la langue la conduit à décrire ceux-ci avec un degré de liberté considérable. Bottineau (2010) précise que la faiblesse de ces théories ne se situe pas nécessairement au niveau du manque de contraintes en soi, la principale faiblesse de ces théories réside pour lui sur le plan de leur inspiration vériconditionnelle :

(...) les acceptions des lexèmes y sont en effet constamment définies par rapport aux conditions du monde, mais conceptualisées; ce qui introduit la question de la spécificité de ces acceptions et de leur structure catégorielle. Dans cette optique, en effet, la description des signifiés n'est pas contrainte du point de vue linguistique (...). Nous sommes donc en présence d'une approche définitionnelle et sémasiologique du signifié, qui cherche à identifier le signifié hors de tout contexte et à le pourvoir d'un contenu recouvrant à la fois des effets sémantiques possibles dans tout contexte, et les connaissances sur le monde qui l'enveloppe. (Bottineau, 2010 : 14) [nous soulignons]

Il est légitime de s'interroger sur la possibilité effective de « définir » ainsi le signifié dans une perspective holistique et d'en saisir un invariant, prédictible en tout contexte, eu égard aux connaissances du monde associées à ce signifié, qui en «captureraient » une signification, dès lors stable, voire non modifiable. Mais cette recherche, si ambitieuse soit-elle, considère tout ce qui se rattache au signifié et au signifié seul. On s'interroge sur ce qui préside à l'émergence idéelle de ce signifié et sur ce qui le rend effectivement concevable. Autrement dit, nos questionnements se tournent vers le sujet transcendantal, et plus encore, ils s'orientent vers le sujet vivant, un sujet percevant inscrit dans un corps, un sujet doté d'une expérience sensorimotrice qui évolue dans un monde qui se présente plutôt comme un arrière-plan - un cadre, un champ qui englobe l'ensemble de notre expérience, mais qui ne se laisse pas saisir en dehors de notre structure, de notre comportement, et de notre cognition. (Varela et al, 1993 : 203).

Si les recherches sémantiques, et notamment la sémantique cognitive, essaient de se tenir au plus près de la problématique du sujet transcendantal, des nuances au demeurant sibyllines n'ont, pour l'heure, pas été réellement explorées. C'est pourquoi nous abordons une deuxième lecture de nos données dans la présente partie, qui met au jour des problématiques encore absconses aujourd'hui, problématiques qui nous font nous détacher du paradigme cognitiviste pour adopter un point de vue enactiviste pour répondre, au mieux, aux questions soulevées. Cette perspective enactive nous rapproche encore davantage du sujet vivant, du sujet percevant, d'un sujet parlant dont la 
production langagière filtre les traits de la réalité ou plutôt d'une réalité, qui représente pour le sujet une pertinence dans le hic et le nunc de la situation dans laquelle il s'inscrit.

\section{2. Expériences statique et dynamique : quelle incidence sur les emplois prépositionnels?}

La présente expérience propose aux participants de suivre un trajet, leur imposant des contraintes similaires, dictées par un protocole analogue; certains étudiants proposant une description "statique " tandis que l'on recueille des descriptions "dynamiques " d'un second groupe d'étudiants (cf. 1.2.2.). L'expérience corporelle statique ou dynamique, en amont des descriptions langagières énoncées par les sujets, représente ici le trait à partir duquel on propose une relecture des données empiriques. Pour ce faire, les comptes rendus recueillis par les participants sont comparés de la manière suivante : les descriptions françaises dont les locuteurs ont fait une expérience dynamique en amont de la tâche descriptive requise sont comparées aux autres échantillons français dont les sujets interrogés sont restés dans une posture corporelle stationnaire. Un rapprochement similaire est effectué avec les descriptions anglaises afin de mener une étude parallèle des données françaises et anglaises, dans l'objectif de faire ressortir des observables qui se manifestent difficilement à la simple lecture de la totalité des descriptions collectées.

\section{2. 1. Emplois prépositionnels récurrents en français}

Parmi les 25 descriptions françaises, la distribution des prépositions et locutions prépositionnelles recensées ont été classées selon leur indice de fréquence dans les 12 descriptions correspondant aux descriptions «statiques » et les 12 autres comptes rendus cadrant avec la problématique « dynamique ». Une première remarque s'impose quant à la distribution des unités prépositionnelles employées lors des deux types d'expériences. Comme mentionné supra, les unités les plus employées par les locuteurs interrogés concernent les items que Melis identifie comme "prépositions simple » ou prépositions «typiques» (Melis, 2003: 105). Les unités les plus récurrentes dans les données correspondent effectivement à celles que Melis classe parmi les plus récurrentes dans le français fondamental ainsi que dans certains corpus attestés ${ }^{28}$. On retient, parmi les items les plus fréquents - et sans tenir compte du type d'expérience effectuée par les locuteurs - un emploi important de à, de, et avec, ce qui corrobore les statistiques sur l'indice de fréquence desdits items, statistiques basées sur les données du français fondamental et sur celles des corpus FRANTEXT et GEOPO (Vaguer, $2008: 22$ ).

L'occurrence des prépositions les plus fréquentes ne constitue pas directement l'objet de notre propos, notre attention étant davantage attirée par un phénomène directement lié à l'emploi plus ou moins conséquent de certaines prépositions suite aux expériences spécifiques auxquelles chaque locuteur souscrit. Effectivement, après l'expérience statique proposée aux participants, on observe un emploi largement supérieur des prépositions au milieu de, autour de, entre et derrière. En contrepartie, l'emploi de ces mêmes prépositions s'avère bien moindre après l'expérience dynamique effectuée par les sujets parlants, comme le montre le tableau $\mathrm{n}^{\circ} 8$ : 
Tableau 8 - emplois prépositionnels français suite aux deux types d'expérience

\begin{tabular}{|l|l|l|l|l|l|l|l|}
\hline \multicolumn{4}{|l|}{ Expérience statique } & \multicolumn{4}{|l|}{ Expérience dynamique } \\
\hline au milieu de & derrière & entre & autour de & au milieu de & derrière & entre & autour de \\
\hline 33 & 28 & 25 & 18 & 9 & 12 & 11 & 7 \\
\hline
\end{tabular}

88 Concernant l'emploi de chaque unité, on observe d'abord que la locution prépositionnelle au milieu de est largement employée suite à une expérience statique (cf. 33 emplois). La notion d'intériorité qu'elle suggère s'avère davantage marquée par dans dont les occurrences sont réparties dans les descriptions dynamiques (cf. 12 occurrences); cette notion d'intériorité reste toutefois moins exprimée dans les comptes rendus dynamiques. Quant aux trois autres items prépositionnels amplement utilisés dans les récits statiques (i.e. derrière, entre et autour de), aucune autre préposition équivalente ne fournit de substitut lexical dans les échantillons dynamiques, par rapport à leur emploi considérable dans les descriptions statiques.

D'une manière parallèle, une étude approfondie des prépositions recensées dans les représentations langagières dynamiques mettent au jour un emploi supérieur des prépositions au fond de, jusque, le long de et vers, comparé à l'insertion des mêmes items dans les descriptions statiques, comme le signale le tableau ${ }^{\circ} 9$ :

Tableau 9 - emplois prépositionnels français suite aux deux types d'expérience

\begin{tabular}{|l|l|l|l|l|l|l|l|}
\hline \multicolumn{2}{|l|}{ Expérience statique } & \multicolumn{4}{|l|}{ Expérience dynamique } \\
\hline jusque & le long de & vers & au fond de & jusque & le long de & vers & au fond de \\
\hline 18 & 12 & 13 & 8 & 35 & 34 & 28 & 21 \\
\hline
\end{tabular}

Une relecture des données se rapportant aux descriptions statiques ne fait pas ressortir d'items prépositionnels, nominaux ou adverbiaux qui pourraient équivaloir à la justesse sémantique suggérée par le sémantisme desdites prépositions ou locutions prépositionnelles dans les comptes rendus dynamiques.

91 Dans les deux cas de figure présentés dans les tableaux $n^{\circ} 8$ et $n^{\circ} 9$, les locuteurs font usage des mêmes unités prépositionnelles, indépendamment des types d'expérience vécues en amont des descriptions requises. Ce résultat met en relief une certaine uniformité langagière relative aux emplois prépositionnels. En revanche, l'emploi accru de quatre unités particulières après chaque type d'expérience démontre la pertinence de localiser certaines entités par rapport à d'autres par lesdites unités selon un groupe d'individus et, à l'inverse, l'absence de pertinence perçue par un deuxième groupe d'individus; les deux groupes assistant à des phénomènes relevant de stimuli oculomoteurs identiques mais livrant une description desdits phénomènes après avoir été conditionné physiquement par des expériences corporelles différentes.

92 Une analyse précise des occurrences spécifiquement concernées par ces emplois inégaux de prépositions signale la caractéristique sémantique suivante: les prépositions et 
locutions employées en priorité après une expérience dynamique révèlent la notion de directionnalité : effectivement, au fond de, jusque, le long de et vers présentent un trait de direction dans les échantillons examinés. Ces items s'avèrent employés par des locuteurs dotés d'une vision binoculaire frontale monodirectionnelle (Georgescu et Goilan-Sandu, 2007 : 96) suivant le parcours d'une entité qu'ils localisent en sélectionnant lesdits items, comme dans les occurrences (14) et (15) :

(14) ${ }^{18} \mathrm{Il}$ refait le chemin jusqu'au bout de la pièce. [FR, 04]

(15) ${ }^{7}$ Elle revient vers le cerceau de départ. [FR, 02]

La notion de direction se manifeste en partie ${ }^{29}$ par les items jusque et vers des occurrences (14) et (15). On note, en revanche, que le domaine de la direction ne ressort pas de l'emploi des prépositions et locutions retenues en priorité par les locuteurs ayant eu une expérience statique. En effet, au milieu de, autour de, entre et derrière ne manifestent pas la même attention du contexte spatial de la part des locuteurs et s'opposent d'ailleurs à la directionnalité que suggèrent les unités précédemment commentées; derrière représentant la trace sémantique la plus emblématique de cette différentiation attentionnelle manifestée par les locuteurs. Si les quatre unités qui nous préoccupent n'ont pas de lien effectif avec la directionnalité, elles renvoient toutes néanmoins au type de prépositions qui sollicite un partage triparti (tripartite scene partitioning) de la scène donnée, pour localiser la figure par rapport au fond (Talmy, 2000a: 313). L'occurrence (16) exemplifie l'un de ces emplois :

(16) ${ }^{6}$ Puis il marque un temps d'arrêt au milieu de la pièce. [FR, 6]

Au milieu de est ici employé dans une occurrence qui marque l'absence de mouvement (cf. marque un temps d'arrêt). En référence aux tableaux $n^{\circ} 8$ et $n^{\circ} 9$, qui mettent en avant les prépositions davantage employées suite à des expériences statiques et dynamiques, on note au total, pour 12 participants réalisant l'expérience statique, 104emplois de au milieu de, autour de, entre et derrière suite à une expérience statique (comparé à 39 emplois des 13 participants effectuant une expérience dynamique), et 118 emplois de au fond de, jusque, le long de et vers, après l'expérience dynamique accomplie par 13 sujets (comparé à51 emplois des 12 individus ayant réalisé l'expérience statique).

\section{2. 2. Emplois prépositionnels récurrents en anglais}

Les 25 descriptions anglaises ont été examinées parallèlement aux comptes rendus français, autrement dit, les prépositions des13 récits faisant suite à une expérience dynamique ont été comparées aux unités relevées dans les 12 descriptions livrées en amont d'une expérience statique. On rappelle que les descriptions anglaises comptent au total 474 propositions dont $74,5 \%$ d'entre elles contiennent au moins une préposition. On observe, suite aux expériences statiques proposées aux locuteurs, que trois prépositions et locutions prépositionnelles se distinguent; parmi elles between, in the center of et past, comme dans l'occurrence (17):

(17) ${ }^{20}$ She stands still between the yellow path and the blue path. [АВ, 36]

On note également un emploi assez conséquent de around, alternativement employé en tant que préposition et en tant que satellite. On relève d'ailleurs davantage d'occurrences de around en tant qu'adverbial phrase (e.g. ${ }^{20} \mathrm{He}$ is turning around. [AB, 27]), comme l'indique le tableau $\mathrm{n}^{\circ}$ 10. En revanche, on ne recense des trois unités juste citées (between, in the center of et past) que des emplois prépositionnels. 
Tableau 10 - emplois prépositionnels anglais suite aux deux types d'expérience

\begin{tabular}{|l|l|l|l|l|l|l|l|l|l|}
\hline \multicolumn{1}{|l|}{ Expérience statique } & \multicolumn{5}{l|}{ Expérience dynamique } \\
\hline between & $\begin{array}{l}\text { in the center } \\
\text { of }\end{array}$ & past & around & around & between & $\begin{array}{l}\text { in the center } \\
\text { of }\end{array}$ & past & around $^{\mathrm{S}}$ & around \\
\hline 48 & 38 & 35 & 13 & 11 & 22 & 21 & 16 & 6 & 9 \\
\hline
\end{tabular}

97 S: around employé comme Satellite

$98 \mathrm{p}$ : around employé comme Préposition

99 Comme cela a été observé dans la langue française, les locuteurs anglais font particulièrement usage de la notion de centralité dans les discours statiques, notamment par between et in the center of. Des descriptions dynamiques ressortent peu d'occurrences prépositionnelles ou satellitaires permettant de marquer, de manière analogue, la notion de centralité, massivement sollicitée dans les comptes rendus statiques. Si on relève quelques mentions du satellite in between dans les récits dynamiques pour véhiculer un sens équivalent à [between $+S N$ ], la récurrence de cette unité est bien moindre comparé à la fréquence de la formule [between $+S N$ ] dans l'autre groupe de récits pour exprimer la notion de centralité. Quant à past et around, les caractéristiques géométriques de ces deux items sont larges en ce sens que leur emploi n'attire pas l'attention sur une identification topologique caractéristique, comme le souligne Svorou, commentant l'emploi de around :

Motions such as AROUND are prototypically understood by reference to [objects] with apparent elongated side, spherical objects, concave and transversal objects, or, finally, objects with prominent parallel boundaries, which we may cross. (Svorou, 1994 : 28)

100 Svorou décrit les entités auxquelles se rapporte around comme des objets dotés de configurations géométriques bien différentes, ne centrant donc pas l'occurrence de l'unité prépositionnelle ou satellitaire sur une pertinence topographique précise.

101 Parallèlement à l'examen des discours statiques, les prépositions et satellites particulièrement employés dans les descriptions dynamiques ont de même été explorées. L'emploi plus important de trois d'entre elles ressort des descriptions, à savoir celui de to, toward et along, comme le signalent les statistiques du tableau $\mathrm{n}^{\circ} 11$.

Tableau 11 - emplois prépositionnels anglais suite aux deux types d'expérience

\begin{tabular}{|l|l|l|l|l|l|}
\hline \multicolumn{3}{|c|}{ Expérience statique } & \multicolumn{3}{l|}{ Expérience dynamique } \\
\hline to & toward & along & to & toward & along \\
\hline 58 & 32 & 28 & 72 & 54 & 48 \\
\hline
\end{tabular}

102 En comparaison aux tableaux $n^{\circ} 8,9$ et 10 affichés supra, le tableau $n^{\circ} 11$ représente celui qui expose un contraste quantitatif moindre entre les prépositions utilisées lors de l'expérience dynamique et les mêmes unités en contrepartie employées suite à un conditionnement physique quasi-immobile des sujets. Effectivement, la préposition la 
plus employée est to, avec 72 occurrences en contexte dynamique mais également 58 emplois en contexte statique. L'écart d'occurrences entre les deux expériences s'avère plus notoire pour toward (i.e. $54>32$ ) et along (i.e. $48>28$ ) même si les trois items restent particulièrement employés suite aux deux d'expérience. On observe une fréquence particulière de l'emploi d'autres prépositions et satellites comme at ou encore back, mais leur fréquence étant particulièrement élevée suite aux deux types d'expérience, l'écart d'emplois desdites prépositions ne présente aucune pertinence et reste sans objet pour la question qui nous préoccupe ici.

Concernant les trois prépositions ici commentées, to et toward identifie toutes deux selon Langacker la notion de $"$ but $»^{30}$. Compte tenu du contexte spatial dans lequel sont employées ces prépositions, le but dont il est ici question correspond souvent aux différentes destinations que doivent atteindre les participants, pour y déposer divers types d'entités (cf. tableau $\mathrm{n}^{\circ} 1-1.2$.) [e.g. Vous irez déposer ces balles dans le cerceau orange situé à côté du parapluii $e^{31}$. Along représente une préposition particulièrement récurrente pour se référer aux différents chemins qu'il incombe aux locuteurs de décrire [e.g. ${ }^{8} \mathrm{After}$ walking along the bluepath (...)]. En dépit de la fréquence des trois unités, à l'issue des deux types d'expérience, leur occurrence ressort particulièrement dans les descriptions faisant suite à une expérience dynamique ; ces éléments insistant - comme en français - sur la notion de directionnalité.

Dans la même veine, il ressort des descriptions suite à une expérience statique, des prépositions n'étant pas autant orientées d'un point de vue topologique que le sont les prépositions signalant la directionnalité. En revanche, comme on l'a constaté en français, ces prépositions - between, in the center of, past et around - représentent les unités dont Talmy identifie, par leur emploi, une distribution tripartie d'un point de vue topographique pour repérer l'élément localisé (i.e. la figure) par rapport à l'élément localisant (i.e. le fond). Par conséquent, on observe que se manifestent, dans les deux langues, et suite à des expériences sensorimotrices similaires, des groupes de prépositions qui semblent présenter la même saillance sémantique aux sujets parlants interrogés. Autrement dit, qu'ils soient francophones ou anglophones, les locuteurs sélectionnent certaines prépositions suggérant un/des trait (s) qui leur semblent identifier une pertinence semblable après avoir souscrit au même type d'expérience physique.

Ce résultat nous permet donc d'établir un lien entre l'emploi d'unités lexicales, en l'occurrence, prépositionnelles et l'activité corporelle qui a présidé à l'émergence desdites unités. Ce constat survient dans le prolongement des questionnements typologiques qui opposent le français et l'anglais. Mais les présentes observations s'éloignent du rapport typologique de la langue tel que l'invoque la lecture talmienne, puisque nos remarques se basent sur les conditions d'applicabilité effectives des items prépositionnels, ce qui situe alors notre prise de vue théorique sur un plan sémantique, c'est en tout cas le premier angle que convoquent ces statistiques en deuxième lecture des données. Pour tenter d'expliciter les similitudes inter-langagières constatées, nous proposons, à partir de l'approche de la sémantique cognitive, de compléter ce paradigme, par une perspective théorique basée sur l'enaction, qui se tient au plus près du sujet parlant, qui est avant tout un sujet vivant. 


\section{3. L'expérience verbale}

106 L'un des objectifs de ce travail consiste à mesurer l'incidence putative de l'activité motrice envisagée comme facteur permettant de promouvoir la construction du sens de la production langagière. Ce type d'investigation n'adopte donc pas une thèse représentationaliste comme celles qui identifient le signifié à une représentation. On peut en effet étudier le traitement cognitif qui préside à l'application des prépositions spatiales sans pour autant identifier les représentations qui interviennent dans ce traitement au signifié des prépositions.

Or, le signifié d'une expression et la conceptualisation qui préside à son application possible sont considérées comme équivalents dans le paradigme cognitif, ce qui nous autorise à nous interroger comme suit: comment la représentation des conditions d'applicabilité peut-elle être confondue avec le signifié et surtout, comment le signifié peut-il ainsi être réduit à la représentation qui le préside ? Ces questions s'adressent aux théories qui ne font pas nécessairement de distinction entre signifié et représentation ${ }^{32}$. Ces théories, d'une certaine manière, justifient les hypothèses ici énoncées à partir de la présente expérience commentée, dont les statistiques recueillies nous détournent de la thèse représentationaliste et orientent nos explications sur le plan expérientiel dans lequel le locuteur s'inscrit.

\section{3. 1. L'expérience préverbale}

Si nous choisissons de ne pas faire coïncider signifié avec représentation, nous défendons la thèse selon laquelle "tout organisme vivant consiste à s'auto-constituer dans son rapport à son monde; à produire sa clôture opérationnelle dans l'action même par laquelle il « configure" son monde. » (Penelaud, $2010: 4$ ). Penelaud promeut un système selon lequel il nous faut concevoir un système, dont les résultats des processus sont des processus eux-mêmes, comme le précisent Varela et al. :

La notion de clôture opérationnelle est une manière de spécifier les classes de processus qui, dans leur fonctionnement proprement dit, se retournent sur euxmêmes pour former des réseaux autonomes. Ces réseaux se rangent [...] dans la classe des systèmes spécifiés par des mécanismes internes d'auto-organisation. (Varela et al, $1993: 200$ )

Varela et al. promeuvent ainsi l'idée de processus automodificateurs, ce qui signifie que l'on ne peut séparer ces processus des produits qu'ils engendrent. Cette activité consiste donc à effectuer, en permanence, des modifications de soi. Ce paradigme place le sujet au centre de cette nouvelle conception du monde et nous oblige à nous défaire de l'idée d'un monde indépendant, conçu comme inséparable de la structure de ces processus d'automodification (Ibid. : 199). "Nous devons mettre en cause l'idée que le monde est pré-donné et que la cognition est représentation. » (Ibid. : 200). Nous défendons ici un modèle dans lequel le terme « représentation » n'est pas ici à prendre dans son acception commune en sciences cognitives, mais au sens premier de son étymon latin : repraesentare comme « rendre présent ». C'est cette définition de la représentation que nous proposons ici d'explorer pour comprendre au mieux les faits langagiers collectés.

110 Aussi, pour être en capacité d'expliciter la sélection des prépositions recueillies qui représentent la trace langagière de surface, il nous parait essentiel d'investiguer en premier lieu l'expression préverbale. Le stade pré-langagier situe le sujet dans une 
approche perceptive, qui apparaît comme un processus actif de formation d'hypothèses. Il importe au sujet de transmettre un message doté d'une signification, vectorisée par la parole. Sur le plan conceptuel, ce projet de signification est constitué d'une synthèse synchronique d'actes langagiers antérieurs et de l'objectif discursif imminent.

Ce faisant, le sujet est conscient de devoir conformer la conceptualisation qui est la sienne au protocole linguistique de "la langue» pour en modéliser le contenu. Effectivement, parler dans une langue donnée à autrui, c'est lui faire produire une présentation sémantique en improvisant dans le cadre des normes syntaxiques, lexicales, morphologiques et prosodiques prévues par le système propre à cette langue. La phase qui précède la parole consiste naturellement en une pensée non verbale. Mais pour comprendre l'enchaînement de ces deux étapes et ainsi tenter de mieux saisir l'influence et le rôle des stimuli corporels qui ont manifestement une incidence sur l'élaboration du sens véhiculé par la langue, nous proposons une description simplifiée de l'expérience en général.

\section{3. 2. Sélection - perçaction - enaction : tentative d'élaboration sémantique}

\section{3. 2. 1. Sélection - perçaction}

112 La confrontation du sujet au monde, préalablement à sa production linguistique, suscite des actes de perceptions qui consistent à élaborer des scènes mentales traitant de signaux captés par les récepteurs sensoriels du sujet (Bottineau, 2011e: 20). L'expérience de l'individu contribue à façonner l'interprétation d'une réalité, qui ne reflète pas une réalité ontologique "objective", mais qui concerne exclusivement la mise en ordre de l'organisation d'un monde, constitué par l'expérience du sujet dans le hic et le nunc de la situation dans laquelle il s'inscrit. (Von Glasersfeld, 1998 : 22). Le sujet croit « percevoir le monde» et produire une transfiguration mentale d'un monde matériel objectif préexistant, or le monde physique «objectif » auquel est confronté tout être vivant est une inconnue, un X-monde (Bottineau, $2010: 17$ ).

Par rapport à ce "monde», propre à chacun, la condition du vivant n'est pas la perception mais ce que Berthoz nomme la perçaction (Berthoz et Andrieu, $2008: 10$ ), à la suite de la philosophie du corps développée par Merleau-Ponty. La perçaction doit être comprise comme l'invention d'un spectacle à partir d'observations du sujet, observations dont la structure et le contenu sont déterminées par le potentiel d'interactions de ce corps au monde, et surtout, par le bilan actionnel déjà vécu par le sujet dans son expérience antérieure (Bottineau, 2012e: 4). L'art de percevoir représente ce à quoi l'organisme est confronté et ce qu'il expérimente en tant que "réalité». Le sujet empirique crée cette réalité, par conséquent, lors du processus que constitue la perçaction, l'organisme, en même temps qu'il extrait les éléments qu'il juge pertinents pour et par son activité, prélève des données qui ne révèlent pas que de traits pertinents, mais qui dégagent aussi des contraintes et des limites. Aussi notre cognition du réel se manifeste elle-même uniquement là où nos constructions échouent (Korzybski, 1998 : 20-43).

L'art de percevoir n'est pas celui de se figurer ce qui existe, mais d'extraire du chaos ambiant quelques éléments à partir desquels le corps compose une synthèse irréaliste, incomplète, et partielle, mais pragmatique et efficace, orientée vers la possibilité de réagir et d'agir. Autrement dit, la perception façonne continuellement un monde modèle (et non un modèle du monde), un modèle dynamique simplifié, ordonné, hiérarchisé par l'action et en vue de l'action (Bottineau, 2012e : 4). Ce modèle émerge par le processus de 
sélection d'une partie infime des signaux disponibles par les différents capteurs du sujet, sélection qui donne lieu au traitement desdits signaux. Ce traitement est conditionné par les états physiques, émotionnels, perceptifs et mentaux du sujet, et constituent les influences créés par le sujet-même, «qui choisit, dans le monde physique, les stimuli auxquels il sera sensible» (Varela et al. , 1993: 236). Cette observation fait écho à la citation de Merleau-Ponty: " Ainsi, la forme de l'excitant est créée par l'organisme luimême. » (Merleau-Ponty, 1942: 12).

\section{3. 2. 2. Enaction}

La perçaction fait l'économie de la notion de représentation en considérant que la synthèse du perçu entremêle au traitement du signal tous les éléments de connaissance issus des acquis de l'engagement moteur au monde (...) : la signification des objets, le sens des choses perçues fait en réalité partie du montage intrinsèque réalisé par le processus perçactif. (Bottineau, 2012e : 5) [nous soulignons]

Bottineau souligne le processus du perçu comme indépendant de la notion de représentation. Le processus complexe de la perçaction nous fait agir et réagir dans ce «monde produit» ou enacté dans le cadre de la théorie de l'enaction de Varela et Maturana. L'attention n'est plus portée ni sur le sujet ni sur l'objet, considérés comme initialement et simultanément co-présents, mais sur le processus qui les lie (Penelaud, 2010 : 6). La phénoménologie, revendiquée par Varela, se caractérise par sa dimension opératoire et procédurale. Dès lors, le sujet percevant, inscrit dans un corps, ne représente plus un monde préétabli, mais il enacte [de l'anglais, to enact : susciter, faire advenir, faire émerger] un monde par l'histoire du couplage structurel qui le lie à son milieu (Varela et al, 1993 : 272). L'approche de la perception par l'enaction envisage la cognition, non comme représentation, mais comme action incarnée (Thompson, 2007 : 13-22) :

Nous voyons au milieu du monde, parce que notre esprit est autant dans le monde que le monde est dans celui-ci, par le flux constant de leur codétermination et de leur recommencement perpétuel, c'est-à-dire : leur enaction. (Penelaud, $2010: 9$ )

Puisque les représentations ne jouent plus de rôle central, ces observations reconsidèrent le statut de l'environnement comme source d'entrées qui se retire « à l'arrière-plan »:

Le monde se présente plutôt comme un arrière-plan - un cadre, un champ qui englobe l'ensemble de notre expérience, mais qui ne se laisse pas saisir en dehors de notre structure, de notre comportement, et de notre cognition. De ce fait, ce que nous disons à propos du monde, en dit au moins autant sur nous-mêmes que sur le monde. (Varela et al, 1993 : 203)

118 Ainsi, le monde produit est d'autant plus acceptable comme réel pour le sujet que toute action dans l'X-monde va se traduire par la réintroduction dans « son » monde produit de perturbations prévisibles et conformes à un système d'attentes et d'intentions (Bottineau, 2010 : 17). Les individus munis du même système sensorimoteur partagent une procédure de production commune et arrivent à des mondes enactés analogues.

\section{4. De la perçaction à l'expérience verbale : débat épistémologique}

119 Dans le cadre d'une approche enactive de la dynamique de la parole (Bottineau 2010, 2011), on cherche à comprendre l'opération de construction sémantique que cette approche active au moment où elle survient dans la linéarité. Cette perspective interroge les dimensions processuelle et expérientielle de la construction du sens ; expérientielle car 
la présente expérience semble démontrer que l'expérience sensorimotrice s'inscrit dans la trace langagière que manifeste la parole. Celle-ci représente la mise en œuvre d'une technique cognitive incarnée, située, enactive, distribuée et interactive. Dans la présente expérimentation, il n'est pas question de réduire la parole à un rôle instrumental ou à une fonction pragmatique spécifique. La parole est l'un des moteurs incarnés de la dynamique psychologique humaine individuelle et collective.

Si elle manifeste la création enactive d'une présentation cognitive collective, il ne convient pas pour autant de nier la part d'internalisation qu'elle sous-tend. Par le paradigme enactif que l'on propose ici, il ne s'agit pas de faire équivaloir au terme d' internalisation le phénomène de représentation, vocable inadéquat comme le signalent les observations mentionnées supra, et terme captieux pour mettre au jour les effets des conditions matérielles d'expérienciation de la motricité sur l'inscription prépositionnelle des descriptions recueillies. Dans l'objectif d'évaluer l'expérience vécue par le sujet dans ses emplois prépositionnels, nous ne défendons pas la thèse d'une représentation de l'expérience du sujet; nous mettons en avant l'expérience-même, qui, à elle seule nous permet d'appréhender l'accès qu'ont les locuteurs aux occurrences prépositionnelles.

Les résultats exposés dans les tableaux $n^{\circ} 8,9,10$ et 11 signalent une propension parallèle pour l'ensemble des locuteurs interrogés, à faire état de directionnalité après avoir eu une expérience corporelle motrice (cf. tableaux $\mathrm{n}^{\circ} 9$ et 11). En contrepartie, les statistiques obtenues à l'issue d'un maintien postural quasi-immobile chez les sujets manifestent de leur part une appréciation prépositionnelle singulière des objets localisés (i.e. figures)par rapports aux items localisants (i.e. fonds) [cf. tableaux $\mathrm{n}^{\circ} 8$ et 10] : en effet, chaque préposition utilisée révèle, dans les deux langues, une complexité cognitive pour faire état du mouvement ou de la localisation de la figure par rapport au fond, complexité qui n'a pas de réciprocité conceptuelle avec les prépositions employées suite à une expérience motrice, telles qu'elles sont employées par l'autre groupe de sujets.

Effectivement, se rapporter à la direction d'une « entité $\mathrm{A}$ " par rapport à une « entité $\mathrm{B}$ " impose nécessairement un partage binaire de l'espace à partir duquel s'effectue l'occurrence de mouvement ou de localisation, comme avec l'emploi prépositionnel de toward (e.g. ${ }^{9}$ She's zigzaging back toward the purple hoop. [AA, 41]). En revanche, l'emploi de préposition qui impose le partage ternaire d'un espace donné pour situer deux entités requiert de prêter attention à davantage d'éléments dans la scène décrite puisque les entités $\mathrm{A}$ et $\mathrm{B}$ doivent non seulement être conceptualisées comme appartenant au même bloc géométrique mais cet ensemble doit lui-même être réifié par rapport à un autre complexe topologique, comme c'est le cas avec la préposition around (e.g. ${ }^{5}$ She's moving around the hoop $[A B, 46])$. Les résultats démontrent que les sujets apparaissent plus enclins à développer ce déchiffrage attentionnel face à la scène à décrire par un certain type de prépositions (parmi lesquelles around) suite à une expérience statique.

Les statistiques des données recueillies à l'issue des deux expériences sensorimotrices mettent en évidence un lien réel entre la motricité effective de la masse corporelle et l'implication langagière engendrée par celle-ci. On suppose que l'expérience kinesthésique est passible de modifier a posteriori la charge attentionnelle consacrée à un phénomène impliquant lui-même un acte moteur (on fait ici référence à l'acte moteur à décrire impliquant la motricité du deuxième participant de l'expérience). L'acte verbal du sujet ayant déjà parcouru l'itinéraire qu'il décrit semblerait être imprégné par l'expérience motrice vécue par le sujet devenu locuteur, locuteur dont le corps, conditionné par cette expérience, la fait transparaître par les indices prépositionnels 
requis dans l'imminence et qui font écho à la notion de direction (cf. to, toward, vers, jusque ) .

124 La direction, en cette occurrence, semble fonctionner comme un geste mental, comme le partage d'un passé imperceptible qui correspond au souvenir ou à la projection rétroactive de l'expérience vécue. En ce sens, les prépositions véhiculant la notion de direction ressemblent aux fragments de discours dont Bottineau défend la fonction de «méta-madeleine» (Bottineau, 2010: 18): elles convoquent par réminiscence une expérimentation vécue qui se concrétise verbalement. La réminiscence expérientielle de la motricité s'inscrit ici dans le cadre d'un renouvellement des rapports verbaux dans lesquels l'expérience de la direction est enactée linguistiquement.

125 À l'inverse, le sujet s'exprimant à l'issue d'une posture corporelle quasi-immobile semble offrir une disponibilité attentionnelle plus large que ses homologues ayant parcouru l'itinéraire en amont d'en livrer une description, en ce sens que le compte-rendu de l'acte moteur s'avère moins centré sur la directionnalité, comme l'atteste le sémantisme des prépositions employées en masse (cf. between, past, autour de, derrière). Les comptes rendus des sujets ayant eu une expérience quasi-immobile offrent davantage de configurations géométriques qui recrutent des prépositions dont l'interprétation topologique présente la scène décrite sous différents angles, angles qui sollicitent une attention oculomotrice plus souple que les items centrées sur la notion de direction.

Sans preuve scientifique faisant état de la mesure des mouvements oculomoteurs des sujets interrogés dans la présente expérience, nous ne pouvons garantir ces observations, qui, pour l'heure, représentent les constats de l'expérimentation dont la partie précédente (cf. 3.3.) en constitue les prémisses théoriques. Toutefois, si nos observations se confirmaient par des expériences ultérieures, elles apporteraient un élément clé concernant le phénomène de corporéité (ou embodiment). Par corporéité, nous ne faisons pas là référence au phénomène trop souvent employé dans le paradigme cognitiviste comme un phénomène mental binaire, que Hampe dénonce comme le mind-bodydualism, très proche de la thèse représentationaliste (Hampe, $2005: 5)^{33}$. La corporéité que notre expérience met en lumière renvoie à la thèse qui permettrait d'envisager l'activité corporelle comme système d'action participant à la construction du sens, considérant dès lors que l'émergence du sens non pré-donné est inscrite dans la coordination de processus kinesthésiques.

\section{5. Une théorie corporimentale orientée socialement}

Intégrer la corporéité aux avancées des théories linguistiques du paradigme cognitif, une corporéité revue et définie sous un angle enactif, correspondrait à revoir la construction du sens dans une science linguistique renouvelée sur la base d'une empirie élargie à un système d'hypothèses phénoménologiquement contraint. À l'instar de Slobin qui propose de remplacer deux notions clés - la pensée (thought) et la langue (language)- par la paire dérivée bien moins statique - penser (thinking) et parler (speaking) - plus appropriés pour faire ressortir les processus mentaux mis en œuvre durant l'acte de production langagière, nous proposons une hypothèse qui place les processus kinesthésiques au centre d'une orientation théorique, selon laquelle ces processus contribuent à l'élaboration d'un sens non pré-donné et construit par une réminiscence qui se veut avant tout expérientielle. Mais cette réminiscence, loin d'être centrée sur un contenu 
proprioceptif exclusif, est également plurielle et intègre des facteurs hétérogènes (pas nécessairement kinesthésiques) pour façonner la construction du sens.

Les parties précédentes (3.3. et 3.4.) ont permis de détailler le passage de l'acte de perception (cf. perçaction) à celui de la parole par lequel le sujet manifeste la production enactive d'une présentation cognitive collective via une scène sémantique plus ou moins planifiée par et pour lui-même au préalable. Si différents stades retracent le passage de la perception d'un sujet à l'acte de parole dont profite et auquel participe chaque individu, un ultime élément dont il n'a pas ici été fait mention s'ajoute à la thèse corporimentale défendue dans le présent article pour proposer une approche holistique de la construction du sens : il s'agit de l'inscription sociale de l'émergence du sens enacté par le sujet. Les phases de (i) perçaction, (ii)traitement de l'information, (iii) enaction et (iv) expression verbale consignent l'individu et son dispositif corporel biomécanique dans une dynamique sociale - une socialité - qui est fondamentalement incarnée et dans laquelle le sujet parlant est avant tout un sujet social, dont les actions et propos se déterminent en fonction de la projection imaginaire d'un « Tu impersonnel » intériorisé, virtuellement dialogal et témoin, par rapport auquel chacun se positionne par l'action et par le verbe.

Ce « Tu impersonnel » est avant tout intériorisé par le sujet, dans un discours intérieur qui préside à la parole. Cette parole intérieure permet de "penser» dans les termes sociaux normés par les pratiques collectives, qui s'inscrivent dans une dynamique sociale ritualisée par les formes langagières. Cette parole intérieure ou endophasie selon la terminologie de Bottineau (Bottineau, 2010 : 15) constitue la projection imaginaire d'un projet sémantique ciblé, et précède sa diffusion acoustique intentionnelle par l'acte dialogal ou exophasie, parole effective vocalisée qui implique un complexe moteur et sensoriel conjoint (Ibid. : 16). La production langagière, telle que le sujet l'intériorise, s'inscrit inexorablement dans une cognition linguistique sociale, dont le sujet projette (in)consciemment les échos interprétatifs d'un sens enacté par des conditions proprioceptives, émotionnelles, créatives, conjoncturales et souvent contraintes d'une situation discursive pas nécessairement choisie par le sujet.

Dans ce contexte intériorisé de la parole, les résultats de l'expérience commentée dans le présent article nous invitent à considérer l'expérience motrice et sensorielle du dispositif corporel comme jouant un rôle vecteur dans le processus d'intériorisation langagière et dans la production enactive de la dynamique externe de la parole. Ces processus permettent de convoquer, par le vocable prépositionnel, les savoirs associés aux prépositions, interprétées comme fragments réminiscents d'une expérience vécue et enactée verbalement.

\section{Conclusion}

131 La présente étude s'est appuyée sur l'hypothèse selon laquelle l'activité motrice envisagée comme facteur permettant de promouvoir la construction du sens peut influencer la production langagière. Notre recherche s'est basée sur l'une des approches dynamiques de la syntaxe en linguistique cognitive - la typologie talmienne -dont le français et l'anglais exemplifient respectivement les deux profils typologiques. Des corpus oraux ont été réalisés dans le cadre d'une expérience recrutant des locuteurs français et anglais, tous soumis à deux types distincts de contraintes corporelles, avant de décrire des scènes 
les incitant à employer des unités prépositionnelles pour faire état de phénomènes de mouvement et de localisation.

Dans la perspective contrastive du projet, et par les statistiques révélant la fréquence d'emploi des items prépositionnels, qui oppose généralement l'anglais (en tant que langue à satellites) et le français (en tant que langue à cadrage verbal), l'expérience a démontré la conformité des deux langues à la typologie talmienne par l'analyse des descriptions recueillies. La variable non-linguistique insérée dans l'expérimentation, relevant des comportements kinesthésiques proposés aux sujets, n’a pas manifesté de modification quant à la fréquence d'emploi des prépositions, ce qui confirme d'une part (i) le respect de la typologie talmienne dans un contexte oral faisant intervenir plusieurs participants en chaque occurrence expérientielle; ce résultat révélant d'autre part (ii)l'absence de pertinence de la variable non-linguistique dans le cadre de l'évaluation d'une modification éventuelle de la fréquence d'emploi des prépositions en français et/ou en anglais.

Si le facteur sensorimoteur de l'expérience semble maintenir les appartenances typologiques respectives des deux langues, une fois le discours installé en situation interactive, un lien a toutefois pu être établi entre les comportements langagiers des locuteurs et les expériences corporelles sensorimotrices auxquelles ils étaient soumis, plaçant de fait notre argumentation à un deuxième niveau d'analyse, alors dissocié des considérations relatives à la typologie talmienne. Effectivement, dans les deux langues considérées, les locuteurs sélectionnent certaines prépositions suggérant des traits qui leur semblent manifestement pertinents, ce après avoir souscrit au même type d'expérience physique. Ce constat a marqué l'ouverture d'un débat d'ordre épistémologique, relevant, en premier lieu, de l'influence mutuelle des deux phénomènes, l'un langagier, l'autre kinesthésique.

134 Les questionnements qu'imposent ces résultats s'éloignent des réflexions traditionnelles sur la langue, alors examinée dans la suite de ce travail sous l'angle de l'enaction. Dans le cadre d'une approche enactive de la dynamique de la parole, on a cherché à comprendre l'opération d'élaboration sémantique que cette approche active, pour mieux saisir l'incidence de la motricité du dispositif corporel sur la production langagière, et, de fait, mieux appréhender la fréquence de l'emploi parallèle de certaines prépositions utilisées de façon analogue à l'issue d'expériences sensorimotrices identiques. Retracer les stades qui séparent la perception du sujet, son discours intérieur ambiant et la diffusion acoustique vocale d'un message ont révélé l'inscription linguistique d'une expérience en premier lieu sensorimotrice mais également perceptive, créative, intersubjective, distribuée, située et normée d'un individu dont le discours façonne l'interprétation d'une réalité, qui concerne la mise en ordre de l'organisation d'un monde, dans une expérience située dans le hic et le nunc d'une situation donnée.

La corporéité que notre expérience a mise en exergue renvoie à la thèse qui permettrait d'envisager l'activité corporelle comme système d'action participant à la construction du sens, considérant que l'émergence du sens non pré-donné est inscrite dans la coordination de processus kinesthésiques. Le sens véhiculé, comme le dévoile la présente expérience, révélerait alors des traces de réminiscences expérientielles vécues associées à l'acte corporimoteur. L'expérience commentée dans le présent article nous invite ainsi à considérer l'expérience motrice et sensorielle du dispositif corporel comme jouant un rôle vecteur dans le processus d'intériorisation langagier et dans celui de production enactive d'un sens émergent. 


\section{BIBLIOGRAPHIE}

AURNAGUE, M. 2005. « Les Noms de Localisation Interne : tentative de caractérisation sémantique a partir de données du basque et du français ». Cahiers de lexicologie, Didier Erudition, 1996, pp. 159-192. http://artxiker.ccsd.cnrs.fr/artxibo-00000031/document

BARNABÉ, A. 2012. «Corps, perception, déplacements : de l'expérience kinesthésique à la cognition linguistique : étude du schème du chemin en grammaire et sémantique anglaises et statut de ce schème en linguistique cognitive ». Thèse (non publiée) soutenue publiquement à l'université Michel de Montaigne, Bordeaux 3.

BERMAN, R. A. , \& Slobin, D. I. 1994. Relating events in narrative: A cross-linguistic developmental study . Hillsdale, $\mathrm{NJ}$ : Lawrence Erlbaum Associates

BERTHOZ, A. et Andrieu, 2008. " Le corps en acte » dans Psychology. http://

www.psychology.emory.edu/cognition/rochat/lab/sens\%20de\%20soi\%20et\%20sens\%20de.pdf BERTHOZ, A. 2013. La Vicariance. Paris : Odile Jacob.

BORRILLO, D. 2000. L'Homophobie, Paris, PUF, coll. « Que sais-je ?», (ISBN 978-2-13-052179-2)

BORRILLO, A. 2010. « Quand la préposition dans contribue à l'expression d'une relation logicotemporelle de consécutivité », Corela [En ligne], HS-7. URL : http://corela.revues.org/942http:// corela.revues. org/942

BOTTINEAU, D. 2012e. Le langage représente-t-il ou transfigure-t-il le perçu ?. La tribune internationale des langues vivantes, Union des professeurs de langues dans les grandes écoles scientifiques, pp. 73-82. <hal-00770354>

BOTTINEAU, D. 2010h. « Language and enaction ». In J. Stewart, O. Gapenne, et E. Di Paolo (eds), Enaction: toward a new paradigm for cognitive science, 267-306. MIT Press.

BOTTINEAU, D. 2010i. « Les linguistiques cognitives en France, problématiques actuelles ». In A. O. Tchubaryan et E. I. Pivovar (eds). Les sciences cognitives : questions et perspectives, Actes du 1er séminaire franco-russe en sciences cognitives :177-222.

BOTTINEAU, D. 2011e. « Parole, corporéité, individu et société : l'embodiment,entre le représentationnalisme et la cognition incarnée, distribuée, biosémiotique et enactive dans les linguistiques cognitives ». In J. -B. Guignard (dir), Linguistique cognitive : une exploration critique, Intellectica $56,2011 / 12: 187-220$.

BOWERMAN, M. 2007. «Containment, support, and beyond: Constructing topological spatial categories in first language acquisition ». In M. Aurnague, M. Hickmann et L. Vieu (eds.), The categorization of spatial entities in language and cognition, 177-203. Amsterdam : John Benjamins.

Cahiers de linguistique française 13 - théories des actes de langage et analyses des conversations unité de linguistique française - faculté des lettres - Université de Genève - 1992.

Centre National des Ressources Textuelles et Lexicales (CNRTL), crée en 2005 par le CNRS. Pérennité du service et des données garantie par l'adossement à l'UMR ATILF (CNRS - Nancy Université), le soutien du CNRS ainsi que son intégration dans le projet d'équipement d'excellence ORTOLANG. 
DENDALE, P. « Lecture de : Ludo Melis, La préposition en français. », Cahiers de praxématique [En ligne], 44 | 2005, mis en ligne le 01 janvier 2013, consulté le 05 septembre 2015. URL : http:// praxematique.revues.org/1703.

FAGARD, B. 2012. «Côte » dégrammaticalisation - le cas des prépositions. Evolutions en francais, Peter Lang, pp. 87-104, 2008. <halshs-00664689> HAL Id : halshs-00664689 : https://

halshs.archives-ouvertes.fr/halshs-00664689

FAUCONNIER, G. et M. Turner. 2002. The Way We Think. Conceptual Blending and the Mind's Hidden Complexities. New York: Basic Books.

GEORGESCU, C. A. et L. Goilan-Sandu, 2007. « Le couple mouvement-regard dans la psychologie des personnages ». Scientific Publishing and Information Online (SCIPIO), $\mathrm{n}^{\circ}$ 2: 93-98.

GRINEVALD, C. , C. Imbertet A. Söres, 2011. « About Path 'Satellites': Elements for a multi-layered definition of 'Satellites' «. Communication, Atelier « Trajectoire »: AFLiCo n 4, Lyon 2011.

HAGÈGE, C. 2010. Adpositions. Oxford/New York: Oxford University Press.

HAMPE, B. 2005. From Perception to Meaning: Image Schemas in Cognitive Linguistics. Berlin: Mouton de Gruyter.

HEINE, B. 1997. Cognitive Foundations of Grammar. New York/Oxford: Oxford University Press.

HERSKOVITS, A. 1986. Language and Spatial Cognition: An Interdisciplinary Study of the Prepositions in English. Cambridge: Cambridge University Press.

нUмBOLDT,1836 [2001]. On Language. On the Diversity of Human Language Construction and Its Influence on the Mental Development of the Human Species. Cambridge University Press.

IBARRETXE-ANTUNANO, I. 2002. "Linguistic typology in motion events: Path and Manner", http://www.unizar.es/linguisticageneral/articulos/Ibarretxe-ASJU-03.pdf

IBARRETXE-ANTUNANO, I. 2004. « Language typologies in our language use: the case of Basque motion events in adult oral narratives ». In Cognitive Linguistics. Volume 15 (3), 317-349. Copyright c. Mouton de Gruyter.

KORZYBSKI, A. 1998. Une Carte n'est Pas le Territoire. Paris : Éditions de l'éclat.

LAKOFF, G. 1987. Women, Fire, and Dangerous Things. Chicago: The University of Chicago Press.

LANGACKER, R. 1987. Foundations of Cognitive Grammar, vol. 1. Stanford: Stanford University Press.

LANGACKER, R. 1991. Concept, Image, and Symbol: the Cognitive Basis of Grammar. Berlin/New York: Mouton de Gruyter.

LANGACKER, R. 1999. « Assessing the cognitive linguistic enterprise ». In T. Janssen et G. Redeker. Cognitive Linguistic Research: Foundations, Scope, and Methodology, 13-59. Berlin: Mouton de Gruyter.

LANGACKER, R. 1999. « A dynamic usage-based model ». In M. Barlow et S. Kemmer. (ed.). Usage Based Models of Language, 1-63. Stanford, California: CSLI Publications.

LANGACKER, R. 2000. Grammar and Conceptualization. Berlin/New York: Mouton de Gruyter.

LANGACKER, R. 2010. « Reflections on the Functional Characterization of Spatial Prepositions ». In Revue Corela. Disponible sur :

http://corela.edel.univ-poitiers.fr/index. php?id=998.edel.univ-poitiers.fr/index. php?id=998

LE PESANT, D. 2012. Essai de classification des prépositions de localisation.

http://www.shs-conferences.org/articles/shsconf/pdf/2012/01/shsconfcmlf12000114.pdf 
MATSUMOTO, Y. 2011. « Varieties of Path Expressions within and across Languages ». Communication, AFLiCo ${ }^{\circ}$ 4, Lyon, Mai 2011.

MEILLET, A. 1912. « L'évolution des formes grammaticales », in Meillet, A. , Linguistique Historique et Linguistique Générale,131-148. Tome I. Paris : Champion.

MERLEAU-PONTY, M. 1942 [1977]. La Structure du Comportement. Paris : PUF. PAPROCKA-PIOTROWSKA, U. 2012. http://gerflint.fr/Base/Pologne9/paprocka.pdf, Université Catholique de Lublin Jean Paul II, Pologne, pp. 143-163.

PENELAUD, O. 2010. « Le Paradigme de l'Enaction Aujourd'hui. Apports et limites d'une théorie « révolutionnaire ». » PLASTIR 2010/1, 18.

SAPIR, E. 1963. Language. London, Aylesbury: Compton Printing Ltd.

SLOBIN, Dan I. 1996a. « From 'thought to language' to 'thinking for speaking. ». In J. J. Gumperz and Stephen C. Levinson (eds). Rethinking Linguistic Relativity, 70-96. Cambridge: Cambridge University Press.

SLOBIN, Dan I. 1996b. « Two ways to travel: Verbs of motion in English and Spanish ». In Grammatical Constructions: Their Form and Meaning, 195-217. In M. Shibatani and S. A. Thompson (eds). Oxford: Oxford University Press.

SLOBIN, Dan I. 1997. « Mind, Code, and Text ». In J. Bybee, J. Haimanet S. A. Thompson (eds.). Essays on Language Function and Language Type, 437-67. Amsterdam: John Benjamins.

SLOBIN, Dan I. 2003. «Language and Thought Online: cognitive consequences of linguistic relativity ». In D. Gentner et S. Goldin-Meadow (eds). Language in Mind: Advances in the Study of Language and Thought, 157-192. Cambridge: MIT Press.

SLOBIN, Dan I. 2004. " The many ways to search for a frog: Linguistic typology and the expression of motion events ». In S. Strömqvist et L. Verhoeven (eds.) Relating Events in Narrative: vol. 2. Typological and Contextual Perspectives,219-257. Mahwah, N. J: Lawrence Erlbaum Associates Publishers.

SOROLI, E. et al. 2012. Spatial encoding in English and French: Typological constraints on second language acquisition and aphasia, in Language, Interaction and Acquisition Journal. http:// www.umr7023.cnrs.fr/sites/sfl/IMG/pdf/LIA_paper_Soroli_et_al_submitted.pdf

SVOROU, S. 1994. The Grammar of Space. Amsterdam/Philadelphia: John Benjamins Publishing Company.

TALMY, L. 1983. « How language structures space ». In Herbert L. Pick, Jr. , et Linda P. Acredolo (eds). Spatial Orientation: Theory, research, and application, 225-282. New York: Plenum Press. Repris dans Towards a Cognitive Semantics, vol. 1, 2000. MIT Press.

TALMY, L. 2000a. Toward a cognitive semantics: Concept Structuring Systems. Volume. 1. Cambridge, Mass.: MIT Press.

TALMY, L. 2000b. Toward a cognitive semantics:Typology and Process in Concept Structuring. Volume 2. Cambridge, Mass.: MIT Press.

TALMY, L. 2005. « The fundamental system of spatial schemas in language ». In B. Hampe. From Perception to Meaning: Image Schemas in Cognitive Linguistics, 199-234. Berlin: Mouton de Gruyter.

THOMPSON, E. 2007. Mind in Life. Cambridge, Mass.: Harvard University Press.

TRÉSOR de la LANGUE FRANÇAISE informatisé (TLFi). 2003. Dictionnaires XIXE et XXE siècles. Analyse et Traitement Informatique de la Langue Française (ATILF). 
VANDELOISE, C. 2006a. De la Distribution à la Cognition. Paris : L'Harmattan

VARELA, F. , E. Thompson, et E. Rosch. 1993. L'Inscription Corporelle de l'Esprit. Paris : Éditions du Seuil.

VON GLASERSFELD, E. 1998. « Introduction à un constructivisme radical ». In 1998. P. Watzlawick. L'Invention de la Réalité : Contributions au Constructivisme, 19-43. Paris : Éditions du Seuil.

VAGUER, C. 2008. Classement syntaxique des prépositions simples du français. Langue Française, Armand-Colin, 2008, pp. 20-36. <hal-00980412>

https://halshs.archives-ouvertes.fr/hal-00980412/document.

VICTORRI, B. 2010. « Le localisme à l'épreuve du verbe aller ». vol. 8. Corela. Disponible sur :

http://corela.edel.univ-poitiers.fr/index.php?id=1009.

\section{NOTES}

1. Nous entendons par " enactivisme » la théorie issue du paradigme de l'enaction, selon laquelle "Varela propose de considérer que le propre de tout organisme vivant consiste à s'autoconstituer dans son rapport à son monde; à produire sa clôture opérationnelle dans l'action même par laquelle il 'configure' son monde. » (Penelaud, $2010: 4$ ).

2. Ce paradigme place le sujet parlant au centre d'une conception du monde nous invitant à nous défaire de l'idée d'un monde indépendant.

3. Ces modèles langagiers illustrent la théorie de la vicariance [du latin vicarius (i.e. substitut, remplaçant)] (Berthoz, 2013), théorie qui désigne le fait qu'un même objet ou phénomène peut être perçu comme remplissant différents rôles en fonction de nos intentions et de notre Umwelt (Berthoz, 2013 : 30). La typologie talmienne illustre cette théorie sur le plan langagier, soulignant l'accès langagier que le sujet parlant peut avoir du monde à partir de son expérience sensible, comme dans les langues à cadrage satellitaire.

4. Les exemples français et anglais sont extraits de l'article de D. Bottineau (cf. Bottineau, 2010 : 14).

5. La typologie,qui décrit un fonctionnement binaire représenté par deux familles de langues (celle à cadrage verbal et celle à cadrage satellitaire) a été critiquée à divers égards (Victorri, 2010). En effet, de nombreuses langues affichant un fonctionnement typologique spécifique (à cadrage verbal ou satellitaire) présente des emplois verbaux qui ne s'avèrent pas correspondre aux emplois de la famille langagière à laquelle ces langues appartiennent (Victorri note l'exemple français il vole de branche en branche (Victorri, 2010:8)) qui a toutes les caractéristiques d'un emploi verbal issu d'une langue à satellites, or le français est connu pour son appartenance au cadrage verbal). Par conséquent, plutôt que de parler de deux "familles" de langues, nous préférons le terme "tendance », les langues ici analysées étant effectivement marquée par une tendance dominante mais non exclusive, précision qui ne figure pas dans la typologie talmienne. 6. Nous précisons que l'expérimentation à laquelle ont souscrit les participants de la présente étude ont "décrit» des phénomènes de navigation spatiale suite aux consignes qui leur imposaient de rendre compte de ces phénomènes dans un environnementcréé à cette fin, par le truchement d'items linguistiques. Ce faisant, nous sommes conscients de la teneur artificielle du cadre expérientiel de notre étude, que nous souhaitons comparer ultérieurement à des expériences similaires inscrites dans des cadres expérientiels naturels (ex : rue, jardin, centre-ville, etc.), dans lesquels la description de l'espace sera alors pleinement justifiée.

7. Certains sujets ont une expérience "dynamique» (i.e. impliquant une motricité effective) alors que d'autres sujets ne se déplacent pas (cf. expérience «statique ») avant leur description langagière. 
8. Pour plus de détails sur l'ambiguïté catégorielle des satellites, voir Matsumoto (2011) et Grinevald et al. (2011).

9. Nous conservons l'adjectif employé par Slobin dans sa description des "elaborated complex paths": He still wandered on, out of the little high valley, over its edge, and down the slopes beyond (Slobin, 1997 : 439).

10. Les exemples issus des descriptions recueillies sont présentés comme suit: la première indication numérale (ici ${ }^{12}$ ) correspond au numéro de la proposition de la description évaluée (chaque proposition ayant été analysée) ; il s'agit ici de la douzième proposition, chacune d'elle étant numérotée. Suite à l'occurrence figure entre crochets en dernier lieu (i) l'appartenance langagière du sujet enregistré (i.e. Anglais Britannique [AB], Anglais Américain [AA] ou Français [ FR]), et (ii) le numéro du candidat évalué au cours de l'expérimentation. L'expérience ici analysée en compte 50, dont 25 anglophones et 25 francophones.

11. Les éléments figure et ground (Talmy, 2000a, 2000b)correspondent aux éléments trajector/ landmark (Langacker, 1991, 1999 ; Fauconnier \& Turner, 2002), et à la cible et au site (Vandeloise, 2006). Dans la présente étude, on emploiera les termes «figure » et «fond » pour désigner ces deux entités.

12. On pense notamment à l'étude de Svorou sur l'emploi de along et across : Motions, such as ALONG and ACROSS are thus prototypically understood by reference to elongated objects or objects with apparent elongated side, spherical objects, concave and transversal objects, or, finally, objects with prominent parallel boundaries, which we may cross. (Svorou, $1994: 28$ )

13. On s'attache ici à l'étymologie du terme "kinesthésie", apparu d'abord sous sa forme anglaise kinaesthesis (1880), et composé des éléments kin- « mouvement, mise en action » (du grec "je meus ») et -esthésie « sensibilité physique ». [Centre National des Ressources Textuelles et Lexicales (CNRTL)]

14. On regroupe par le terme « occurrences prépositionnelles » les prépositions et satellites dont on fait la distinction, comme indiqué précédemment (cf. 1.3.1.)

15. Cette hypothèse a donné lieu à de nombreuses expériences dans différentes langues du monde, expériences réalisées avec des enfants chargés de décrire les mouvements d'animaux figurant dans un conte pour enfants, ce afin de discerner les différences syntaxiques entre les langues pour faire état du phénomène de mouvement. (cf. Slobin, 1996)

16. On fait ici référence à la prise en compte des satellites et prépositions des énoncés anglais qui contiennent entre un et six satellites dans les descriptions analysées.

17. Ibarretxe-Antuñano, dans son commentaire de l'hypothèse de Slobin, précise la nuance qui distingue les deux hypothèses. En cela, l'hypothèse de Slobin est une version "modifiée ", et Ibarretxe-Antuñano poursuit: "modified version of the classical Sapir-Whorf debate on linguistic relativity, modified because its main goal is not to prove the effects of grammar on worldview or nonlinguistic behaviour, but to show the way in which speakers of a language organise their thinking in accordance to the linguistic tools offered by their native language." (Ibarretxe-Antuñano, 2002: 38).

18. Ce terme anglais est issu des analyses de Langacker, qu'il définit comme une projection dans l'objet même des processus cognitifs utilisés pour le construire (Langacker, 1987 : 262-67). La subjectification constitue une source importante de variation sémantique.

19. Nous insistons sur la désignation "d'un monde», et non pas "du monde ", ce qui nous soumettrait à croire en un monde matériel objectif, potentiellement porteur d'une sémantique universelle que le sujet peut schématiser et que les théories peuvent modéliser. Les précédentes références aux théories de Sapir, de Whorf et de Slobin démentent cet angle de vue, tout comme les considérations théoriques présentées ci-après.

20. On fait ici référence à la définition de Meillet pour identifier le paradigme de grammaticalisation : «Les langues suivent une sorte de développement en spirale : elles ajoutent des mots accessoires pour obtenir une expression intense ; ces mots s'affaiblissent, se dégradent et tombent au niveau de simples outils grammaticaux; on ajoute de nouveaux mots ou des mots 
différents en vue de l'expression; l'affaiblissement recommence et ainsi sans fin. » (Meillet, $1912: 140)$

21. On fait ici référence à la figure et au fond, tels qu'ils ont été définis en première partie (cf. 1.3.2.1.).

22. Le classement desdites prépositions n'étant pas l'objet de notre propos, nous ne commentons pas ce classement dans le présent article. Pour plus de détails, voir Langacker, 2000.

23. Notre propos étant davantage centré ici sur les conditions d'applicabilité des prépositions et satellites d'un point de vue sémantique, on accordera peu d'importance à la distinction préposition vs. satellite d'un point du vue syntaxique, ce qui n'est pas, dans le présent article, l'objectif de notre propos.

24. Les trois références citées qui correspondent aux écrits d'Aurnague et Borillo ne figurent pas dans notre bibliographie.

25. À cet égard, Denis Le Pesant recense en français 186 locutions prépositionnelles (Le Pesant, 2012 : 923).

26. Pour plus de détails sur l'inventaire des différents statuts des prépositions, voir C. Waguer, 2008.

27. à, après, avant, avec, chez, contre, dans, de, depuis, derrière, dès, devant, en, entre, envers, hors, jusque,par, parmi, pendant, pour, sans, selon, sous, sur, vers (Melis, 2003 : 105).

28. On note parmi euX FRANTEXT ou encore GEOPO.

29. Il nous semble essentiel de préciser «en partie " car les items jusque et vers ne peuvent effectivement révéler de direction que par leur inscription au sein d'une occurrence mettant en scène la direction d'une figure par rapport à un fond, et ce, au travers d'un verbe signalant luimême l'expression d'un mouvement (ici, refait le chemin en [14] et revient en [15]).

30. On note à ce propos le classement de ces prépositions que Langacker assimile aux prépositions "1-way", comparées aux prépositions qu'il désigne comme des prépositions «2way». Selon Langacker, chaque préposition peut être interprétée en fonction du schème-image du chemin (cf. Barnabé, 2012), qu'il désigne "source-path-goal"schema pour caractériser chacune d'elles (Langacker, 2000 : 55). Les «1-way» prepositionsreprésentent celles qui coïncident avec les sous-schèmes du chemin et du but (path-goal subschemas),tandis que les "2-way» prepositions correspondent à celles signalées par le datif (Ibid. : 55).

31. Cette instruction correspond à la deuxième des consignes présentées dans le tableau $n^{\circ} 1$.

32. Cette assimilation des deux notions s'affirme, par exemple, par la modalité visuelle pour exprimer le concept chez Langacker.

33. On note la vive critique de Hampe concernant le terme embodiment, trop souvent employé à mauvais escient, et que l'on présume être mal interprété par ses usagers. Pour plus de détails, voir Hampe, 2005.

\section{RÉSUMÉS}

La présente étude s'appuie sur l'hypothèse selon laquelle l'activité motrice envisagée comme facteur permettant de promouvoir la construction du sens peut influencer la production langagière. Notre recherche se base sur la typologie talmienne (Talmy, 2000a). Dans une perspective contrastive, des corpus oraux sont réalisés dans le cadre d'une expérience recrutant des locuteurs français et anglais, tous soumis à des contraintes sensorimotrices avant de décrire 
des scènes les incitant à employer des prépositions pour faire état de phénomènes de mouvement. Une variable liée aux comportements kinesthésiques des sujets nous permettra d'évaluer (i) le maintien de l'appartenance typologique des deux langues considérées, dans un contexte oral faisant intervenir plusieurs participants et (ii) la pertinence de la variable nonlinguistique en tant que facteur contribuant à élaborer le sens. Un lien sera établi entre les comportements langagiers des locuteurs et les expériences sensorimotrices auxquelles ils sont soumis avant de décrire les scènes de mouvement, plaçant de fait notre argumentation à un deuxième niveau d'analyse, alors dissocié de considérations strictement linguistiques. Un débat épistémologique s'ouvrira, relevant de l'influence mutuelle des deux phénomènes, l'un langagier, l'autre kinesthésique. La langue sera alors examinée dans la suite de ce travail, sous l'angle d'un nouveau paradigme, le paradigme enactif.

This investigation relies on the hypothesis that sensorimotor processes are a primary factor in the construction of meaning and therefore influence language production. Our research draws mainly on Talmy's typological framework (Talmy, 2000a) and spoken corpora were collected in an experiment involving English and French speakers. Both groups were subjected to sensorimotor constraints before being asked to describe specific scenes requiring the use of prepositions in order to depict motion phenomena. Based on these results a particular variable bound to the speakers' kinaesthetic processes enables us to assess (i) the robustness of the typological definition of each language in a spoken context with several interactive participants and (ii) the relevance of the non-linguistic variable in the construction of meaning. A correlation will be shown between the speakers' linguistic behaviour and the sensorimotor constraints the subjects were submitted to before depicting the visual scenes. This will allow us to place our argument at a second level of analysis, free from strict linguistic considerations. An epistemological discussion will then be carried out about the mutual influence of the two orders of phenomena, linguistic and kinaesthetic. In the final part of this paper language will be examined under a new paradigm - the enactive paradigm.

\section{AUTEUR}

\section{AURÉLIE BARNABÉ}

Université Blaise Pascal - Clermont-Ferrand

aurelie_barnabe@yahoo.fr 